\title{
Bifurcation and symmetry-breaking
}

\author{
J. Smoller ${ }^{\star \star \star \star}$ and Arthur G. Wasserman $\star \star$ \\ Department of Mathematics, The University of Michigan, Ann Arbor, Michigan 48109, USA
}

\section{§1. Introduction}

Let $E$ and $F$ be Banach spaces, $E \subset F$, and $A$ an interval in $\mathbb{R}$. In this paper we use bifurcation theory to study the set of solutions of an equation $M(u, \lambda)=0$, where $M$ is a smooth operator, $M: E \times A \rightarrow F$. Suppose $G$ is a compact Lie group acting on $E$ and $F$, and that $M$ is equivariant with respect to $G$ in the sense that $M(g u, \lambda)=g M(u, \lambda)$ for all $g \in G, u \in E, \lambda \in A$. Then the set of solutions also admits a $G$-action. Suppose too that $\left(u_{\lambda}, \lambda\right)$ is a smooth curve of invariant (symmetric) solutions under $G$; i.e., $g u_{\lambda}=u_{\lambda}$, for all $g \in G$. We investigate under what conditions there exists bifurcation from this curve of invariant solutions to non-invariant solutions; that is, we study the general problem of "symmetry-breaking". We consider the case where the $u$-derivative, $d M_{\left(u_{\lambda}, \lambda\right)}$ has a finite positive spectrum, and using an equivariant version of the Conley index, we prove the existence of bifurcation whenever there is a "change" in the positive spectrum. This problem arises naturally in the study of bifurcation of radial solutions of the semilinear elliptic equation

$$
\Delta u(x)+f(u(x))=0, \quad x \in D_{R}^{n},
$$

with homogeneous linear boundary conditions

$$
\alpha u(x)-\beta d u(x) / d n=0, \quad x \in \partial D_{R}^{n},
$$

into asymmetric ones (the problem of "symmetry breaking"; see $\left[\mathrm{SW}_{2}, \mathrm{SW}_{3}\right.$, $\left.\mathrm{SW}_{5}\right]$ ). Here $G=O(n)$, the $n$-dimensional orthogonal group, $D_{R}^{n}$ denotes the $n$-ball of radius $R$ centered at the origin, $d / d n$ denotes differentiation in the outwardpointing normal direction, $\alpha^{2}+\beta^{2}=1$, and $f$ is a smooth function. We shall show that our general results apply to this problem, and that under fairly general hypotheses on the nonlinear function $f$, there are infinitely-many symmetrybreaking solutions. Furthermore, we prove that at each such solution there bifurcates out families of distinct solutions having distinct symmetries.

Our general bifurcation result can be described as follows. Suppose that $\left.\left\{\left(u_{\lambda}, \lambda\right)\right\}: \lambda_{1} \leqq \lambda \leqq \lambda_{2}\right\}$ is a smooth family of symmetric solutions of $M(u, \lambda)=0$, and let $P_{\lambda}$ be the vector space generated by the set $\left\{v \in E: d M_{\left(u_{\lambda}, \lambda\right)} v=\mu v\right.$ for

* Research supported in part by the NSF under Grant No. MCS-830123

* Research supported in part by the ONR under Grant No. N0014-88-K-0082 
some $\mu \geqq 0\}$. Then if $P_{\lambda_{1}}$ and $P_{\lambda_{2}}$ are distinct representations of $G$, there is a $\lambda, \lambda_{1}<\lambda<\lambda_{2}$, for which $\left(u_{\lambda}, \lambda\right)$ is a bifurcation point for $M$. To be somewhat more specific, let $M$ be as above, and assume that $P_{\lambda_{i}}, i=1,2$, contains $k_{i}$ copies of some fixed irreducible representation of $G$, where $k_{1} \neq k_{2}$, and that $d M_{\left(u_{\lambda_{i}}, \lambda\right)}$ is non-singular, $i=1,2$. Then modulo two technical assumptions, one of which mimics the notion of ellipticity, and the other of which is valid for groups $G$ having less than 5 components (e.g. $O(n)$ ), we have that bifurcation for $M$ occurs in $\lambda_{1}<\lambda<\lambda_{2}$; (see Theorem 3.3 for the precise statement).

Our method is to construct a "modified", equivariant Lyapunov-Schmidttype reduction, global in $\lambda$, not to the kernel, (or center manifold), as previous workers have done, but to a finite-dimensional subspace containing the spaces $P_{\lambda_{1}}$ and $P_{\lambda_{2}}$. This has the advantage that when e.g., $G=O(n)$, we can obtain the simple condition that the Conley indices (for an associated finite-dimensional problem), are different at $\lambda_{1}$ and $\lambda_{2}$ iff $P_{\lambda_{1}} \neq P_{\lambda_{2}}$, as representations of $O(n)$. This last condition is easily verified in our application to the problem (1.1) (1.2), and enables us to prove that bifurcation must occur. A further argument is given to show that the symmetry breaks.

There are several different ways of relating "infinitesimal bifurcation" to bifurcation, (see e.g. [AZ, B, Ch, K, Kr, R, Ry]), but none of these are applicable to our symmetry-breaking problem, (1.1), (1.2). This is because the problem either lacks a specific form of variational structure, or the hypotheses required of the derivatives are not satisfied, or some "transversality" condition is just too difficult to verify. Moreover, we do not require that eigenvalues be simple, or "odd-dimensional" as required in the bifurcation theorems of CrandallRabinowitz and Krasnoselski, [S]. Our method avoids these difficulties by using the Conley index. Similar ideas were used by Kielhofer [K], who uses the standard Lyapunov-Schmidt reduction (local in $\lambda$ ), to the kernel of $d M_{(u, \lambda)}$, and he also requires a certain "crossing number" to be non-zero. Our approach is simpler, can be done equivariantly, is easily applicable to the symmetrybreaking problem for (1.1), (1.2), and does not require the crossing number to be non-zero.

In Sect. 2 we prove an abstract bifurcation theorem. Our method is to reduce it to one in finite dimensions whereby we show that information on the structure of the spaces $P_{\lambda_{i}},(i=1,2)$, implies that we can explicitly compute Conley indices for rest points of an associated finite dimensional equation. For these equations we prove that bifurcation occurs, and then we transfer this information back to the given infinite dimensional equation. (To apply the Conley Index Theory directly in the infinite dimensional situation is simply too difficult.) These things are discussed in the introduction to Sect. 2 , whereby we also make connections with the results of previous work in the literature.

In Sect. 3 we consider an equivariant form of the Conley index suitable for our purposes. We show how a theorem of Lee and Wasserman, [LW], applies to yield a general bifurcation result in the presence of a group action (Theorem 3.3).

In Sect. 4 we consider the application of our abstract results to the problem (1.1), (1.2). We state our precise hypotheses on $f$, and we summarize the linear 
results contained in $\left[\mathrm{SW}_{5}\right]$. That is, in $\left[\mathrm{SW}_{5}\right]$ we have shown that under rather general assumptions on $f$, "infinitesimal" symmetry breaking must occur, in the sense that there are radial solutions of (1.1), (1.2), with the property that the associated linearized operator about such a solution is singular, and its kernel contains asymmetric elements. We use these type of results in Sect. 5 to show that the hypotheses of our previously obtained general theorems are satisfied, so that bifurcation must occur. In order to rule out some very degenerate situations (which can arise - see [Po]), in certain cases we impose the conditions of analyticity on $f$. Using a general theorem in $\left[\mathrm{SW}_{4}\right]$, we prove that the resulting bifurcating solutions are asymmetric ones, so that symmetrybreaking indeed occurs.

The existence of solutions having particular symmetry groups is discussed in Sect. 6. We prove a general theorem giving conditions under which there exist distinct bifurcating solutions admitting distinct symmetry groups. This is applied to the problem (1.1), (1.2) for subgroups of $O(n)$, the form $O(p) \times O(n-p)$, (and also to other subgroups of $O(n)$ ), and we deduce the existence of bifurcating solutions having at least these symmetries.

Finally in the appendix we show that two apparently different problems are in fact equivalent. Thus as is well-known, we can consider (1.1), (1.2) with fixed boundary conditions and allow the radius $R$ to vary, or equivalently, we can fix the radius and have the equation vary. However, if $\alpha \beta \neq 0$, this requires that the boundary conditions also vary with the parameter. The standard bifurcation framework considers fixed radius and fixed boundary conditions, and only the equation is allowed to vary. That is, if $\alpha \beta \neq 0$, one cannot set up the bifurcation problem in the standard framework as a mapping from a product $B_{1} \times A \rightarrow B_{2}$, where $B_{1}$ and $B_{2}$ are Banach spaces of functions, and $A$ denotes the parameter space. However, we shall show that the relevant function-parameter space forms a vector bundle over $\mathbb{R}$, and is thus locally a product. This local product structure then suffices to fit our problem into the general bifurcation theory framework which we have discussed in the previous sections.

\section{§ 2. An abstract bifurcation theorem}

Let $B_{2}, B_{0}$ be Banach spaces, $H$ a Hilbert space, and assume that $B_{2} \subset B_{0} \subset H$, where each of the embeddings are continuous (in many applications, $B_{2}=C_{\partial}^{2}(\Omega)$, $B_{0}=C^{0}(\Omega), H=L^{2}(\Omega)$ ). Let $A$ be an interval in $\mathbb{R}$, and suppose that $M$ is a smooth operator,

$$
M: B_{2} \times \Lambda \rightarrow B_{0}
$$

We assume too that there is a continuous 1-parameter family $\left\{u_{\lambda}\right\} \subset B_{2}$ satisfying $M\left(u_{\lambda}, \lambda\right)=0$, and we denote by $d M_{\lambda}(w) \equiv d M_{\left(u_{\lambda}, \lambda\right)}(w, 0)$ the associated linearized operators.

Definition 2.1. The peigenspace $P_{\lambda}$ of $d M_{\lambda}$ is the closed sub-vector space spanned by $\left\{v \in B_{2}: d M_{\lambda} v=u \mu\right.$ for some $\left.\mu \geqq 0, v \neq 0\right\}$. The neigenspace $N_{\lambda}$ of $d M_{\lambda}$ is defined similarly but with $\mu \geqq 0$ replaced by $\mu \leqq 0$. (Thus the peigenspace (resp. neigen- 
space) is the space generated by those eigenvectors of $d M_{\lambda}$ corresponding to non-negative (resp. non-positive) eigenvalues.)

In this section we shall show that under mild hypotheses, if $M$ is a gradient and

(*) if for some $\lambda_{1}, \lambda_{2}$ in $\Lambda, \operatorname{dim} P_{\lambda_{1}} \neq \operatorname{dim} P_{\lambda_{2}}$, and both $d M_{\lambda_{1}}$ and $d M_{\lambda_{2}}$ are non-singular, then there exists a $\lambda$ between $\lambda_{1}$ and $\lambda_{2}$ such that $\left(u_{\lambda}, \lambda\right)$ is a bifurcation point for $M$.

Note that if this problem were one in finite dimensions, (i.e., if $B_{2}$ and $B_{0}$ were finite dimensional), then the Conley indices $h\left(u_{\lambda_{i}}\right)$, would satisfy $h\left(u_{\lambda_{1}}\right)$ $\neq h\left(u_{\lambda_{2}}\right)$ so that Conley's Continuation Theorem implies that $(*)$ is valid, (see [S] for details).

Now although the Conley index theory has an extension to the infinite dimensional case, $[\mathrm{C}, \mathrm{S}, \mathrm{Ry}]$, the corresponding notions are quite abstract, and the theory requires some subtle constructions which are not easily applicable to our bifurcation problem. Indeed, in this case it is no easy matter to compute the homotopy types $\left[N_{1} / N_{2}\right]$ of an index pair $\left(N_{1}, N_{2}\right)$, for a non-degenerate solution $u_{\lambda}$, from a knowledge of the peigenspace $P_{\lambda}$, even assuming that $d M_{\lambda}$ is symmetric, and Fredholm of index zero, and that $P_{\lambda}$ is finite dimensional.

In a recent interesting paper, $[\mathrm{K}]$, Kielhofer considers a related problem, and obtains a corresponding bifurcation theorem. His method involves a local argument (in $\lambda$ and $u$ ), reducing the problem to a finite dimensional one on the null space of $d M_{\lambda}$, via a Lyapunov-Schmidt reduction. He uses the idea of a "crossing number", together with a Brouwer-degree argument and certain ideas from the Conley index theory. His result does not apply when the crossing number is zero and indeed, such crossing numbers are often quite difficult to compute. These remarks apply, in particular to the symmetry-breaking problem for (1.1), (1.2). A related result can be found in a paper of Amann and Zehnder, [AZ]; here again the bifurcation problem is reduced to one in finite dimensions, but their result is not applicable to our problem. A similar idea occurs in the paper of Chow and Lauterbach, $[\mathrm{CL}]$, whereby they reduce the problem to a finite-dimensional one on the center manifold; this theory too is not applicable to our problem.

The technique which we use involves a generalized Lyapunov-Schmidt reduction, global in $\lambda$, whereby we reduce the problem to one on a finite dimensional space that contains $P_{\lambda_{1}}$ and $P_{\lambda_{2}}$.

With $B_{2}, B_{0}, H$, and $A$ defined as above, let $M$ be a $C^{1}$ mapping $M: B_{2}$ $\times A \rightarrow B_{0}$, satisfying

$$
M(0, \lambda)=0, \quad \lambda \in \Lambda .
$$

(This entails no loss in generality; merely replace $u$ by $u+u_{\lambda}$.) For $\varepsilon>0$, let $P_{\lambda, \varepsilon}$ denote the peigenspace of $d M_{\lambda}+\varepsilon I$. We assume that there is an $\varepsilon>0$ such that for all $\lambda \in \Lambda$,

$$
\operatorname{dim} P_{\lambda, \varepsilon}<\infty .
$$

Here is the main result in this section. 
Theorem 2.1. Let $M$ be a gradient operator for each $\lambda \in A$, satisfying (2.1), and (2.2). If there exists $\lambda_{1}<\lambda_{2}$ in $\Lambda$ satisfying

(i) $d M_{\lambda_{i}}$ is non-singular, $i=1,2$, and

(ii) $P_{\lambda_{1}}$ is not isomorphic to $P_{\lambda_{2}}$, then there exists a $\lambda_{0}, \lambda_{1}<\lambda_{0}<\lambda_{2}$ such that $\left(0, \lambda_{0}\right)$ is a bifurcation point for $M(u, \lambda)=0$.

We remark that since $P_{\lambda} \subset P_{\lambda, \varepsilon}$, hypotheses (2.2) implies that $\operatorname{dim} P_{\lambda_{i}}<\infty$, $i=1$, 2. Hence condition (ii) means that $\operatorname{dim} P_{\lambda_{1}} \neq \operatorname{dim} P_{\lambda_{2}}$. We have stated (ii) in this slightly awkward way in anticipation of the results in Sect. 3, where we shall extend this theorem to the equivariant case, i.e., to the case where there is a group acting on the space.

Proof. Let $I=\left[\lambda_{1}, \lambda_{2}\right]$. Since $M$ is a gradient, $d M_{\lambda}$ is symmetric, and hence diagonalizable. Since (2.2) implies that the eigenvectors of $d M_{\lambda}$ corresponding to eigenvalues greater than $-\varepsilon$ lie in a finite dimensional space, it follows that there is a finite codimensional space $E_{\lambda} \subset B_{2}$ such that

$$
\left\langle d M_{\lambda} e, e\right\rangle \leqq-\varepsilon|e|^{2} \quad \forall e \in E_{\lambda} \subset B_{2}
$$

where the inner product, and norm are those in $H$. Since the mapping $\lambda \rightarrow d M_{\lambda}$ is continuous (in the strong operator topology), it follows that there is an open interval $I_{\lambda}$ about $\lambda$ on which $\left\langle d M_{\mu} e, e\right\rangle \leqq-\frac{\varepsilon}{2}|e|^{2} \forall \mu \in I_{\lambda}, \forall e \in E_{\mu}$. A finite number of intervals $I_{\lambda 1}, \ldots, I_{\lambda^{s}}$ cover $I$; let $E=\bigcap_{i=1}^{s} E_{\lambda_{i}} \cap P_{\lambda_{1}}^{\perp} \cap P_{\lambda_{2}}^{\perp}$. Then $E \subset B_{2}$ has finite codimension. Note that $E_{\lambda}=P_{\lambda, \varepsilon}^{\perp} \cap B_{2}$, so

$$
E=\bigcap_{i=1}^{s} P_{\lambda_{i}, \varepsilon}^{\perp} \cap P_{\lambda_{1}}^{\perp} \cap P_{\lambda_{2}}^{\perp} \cap B_{2}=\left(\bigcup_{i=1}^{s} P_{\lambda_{1}, \varepsilon} \cup P_{\lambda_{1}} \cup P_{\lambda_{2}}\right)^{\perp} \cap B_{2} \equiv F^{\perp} \cap B_{2},
$$

so that $E$ is a closed subspace of $B_{2}$ of finite codimension. Now if $\mu \in I$, then $\mu \in I_{\lambda, j}$ for some $j$, and if $e \in E$ then $e \in E_{\lambda j}$ so $\left\langle d M_{\mu} e, e\right\rangle\left\langle-\frac{\varepsilon}{2}|e|^{2}\right.$. Thus for each $\lambda \in I, d M_{\lambda}$ restricted to $F^{\perp}$ is uniformly negative definite. Note that $P_{\lambda_{1}} \cup P_{\lambda_{2}} \subset F$.

Now write $H=F^{\perp} \oplus F, B_{2}=\left(B_{2} \cap F^{\perp}\right) \oplus F$, and $B_{0}=\left(F^{\perp} \cap B_{0}\right) \oplus F$, since $F \subset B_{2}$. Let $\pi_{i}$ be the projection $\pi_{i}: B_{i} \rightarrow\left(F^{\perp} \cap B_{i}\right), i=0,2$, and note that $\pi_{0} \circ \pi_{2}$ $=\pi_{2}$. Note too that since $F$ is finite dimensional, and the inner product is a continuous function on $B_{i}, i=1,2$, (since $B_{i}$ is continuously embedded in $H$ ), it follows that $\left(F^{\perp} \cap B_{i}\right)$ is a closed subspace of $B_{i}, i=0,2$.

We are now ready to make a Lyapunov-Schmidt reduction. Thus, for $h \in B_{2}$, we write

$$
\begin{aligned}
h & =(x, y), \quad x \in F^{\perp}, \quad y \in F, \\
M(h, \lambda) & =M(x, y, \lambda)=(u(x, y, \lambda), v(x, y, \lambda)),
\end{aligned}
$$

where $u \in F^{\perp}, v \in F$. Now since $M(0,0, \lambda)=0$ (by (2.2)), it follows that $u(0,0, \lambda)=0$. We claim that $u_{x}(0,0, \lambda)$, (defined on $F^{\perp} \cap B_{2}$ to $\left.F^{\perp} \cap B_{0}\right)$, is an isomorphism for each $\lambda \in I$. To see this, note that $u_{x}(0,0, \lambda)$ is injective since if $u_{x} \xi=0$, for some $\xi \in F^{\perp}$, and $\xi \neq 0$, then as $u_{x}$ is strictly negative definite, the relation 
$\left\langle u_{x} \xi, \xi\right\rangle<0$ gives a contradiction. Next, note that $u_{x}(0,0, \lambda)=\pi_{0} \circ d M_{\lambda}(0,0)$, which we write for short as $u_{x}=\pi_{0} d M$. We use this to show that $u_{x}$ is symmetric; namely for $\pi_{2} y, \pi_{2} z \in B_{2}$,

$$
\begin{aligned}
\left\langle u_{x}\left(\pi_{2} y\right), \pi_{2} z\right\rangle & =\left\langle\pi_{0} d M\left(\pi_{2} y\right), \pi_{2} z\right\rangle \\
& =\left\langle d M\left(\pi_{2} y\right), \pi_{0} \pi_{2} z\right\rangle \\
& =\left\langle d M\left(\pi_{2} y\right), \pi_{2} z\right\rangle \\
& =\left\langle\pi_{2} y, d M\left(\pi_{2} z\right)\right\rangle \\
& =\left\langle\pi_{0} \pi_{2} y, d M\left(\pi_{2} z\right)\right\rangle \\
& =\left\langle\pi_{2} y, \pi_{0} d M\left(\pi_{2} z\right)\right\rangle \\
& =\left\langle\pi_{2} y, u_{x}\left(\pi_{2} z\right)\right\rangle .
\end{aligned}
$$

Next for $x \in H$,

$$
\begin{aligned}
-\frac{\varepsilon}{2}\left|\pi_{2} x\right|^{2} & \geqq\left\langle d M\left(\pi_{2} x\right), \pi_{2} x\right\rangle \\
& =\left\langle d M\left(\pi_{2} x\right), \pi_{0} \pi_{2} x\right\rangle \\
& =\left\langle\pi_{0} d M\left(\pi_{2} x\right), \pi_{2} x\right\rangle \\
& =\left\langle u_{x}\left(\pi_{2} x\right), \pi_{2} x\right\rangle .
\end{aligned}
$$

Thus the spectrum of $u_{x}(0,0, \lambda)$ is uniformly bounded away from zero. Since $u_{x}(0,0, \lambda)$ is symmetric and injective, it follows that $u_{x}(0,0, \lambda)$ is an isomorphism for each $\lambda \in I$; this proves our claim.

Hence by the implicit function theorem, we may solve the equation $u(x, y, \lambda)=0$ for $x=x(y, \lambda)$ in a neighborhood of $(0,0) \times I$. (This holds since we can solve the equation in a neighborhood of $(0,0, \lambda)$ of the form $U_{\lambda} \times 0_{\lambda}$, where $U_{\lambda}$ is a neighborhood of $(0,0)$, and $0_{\lambda}$ is a neighborhood of $\lambda$ in $I$.) By compactness, a finite number of the $0_{\lambda}$ cover $I$, say $0_{\lambda_{1}}, \ldots, 0_{\lambda_{s}}$. Therefore we have a unique solution on $\mathscr{U} \times \mathscr{N} \equiv \bigcap_{i=1}^{s} U_{\lambda_{i}} \times \bigcup_{i=1}^{s} 0_{\lambda_{i}}$. Now define

$$
\phi(y, \lambda)=v(x(y, \lambda), y, \lambda), \quad \lambda \in I .
$$

This is the global Lyapunov-Schmidt reduction which we shall use. We now need two lemmas.

Lemma 2.2. Fix $\lambda \in I$ and consider the differential equation (in finite dimensions), $y_{s}=\phi(y(s), \lambda)$. Then this equation admits a Lyapunov function.

Proof. Write $H=F_{\lambda} \oplus F_{\lambda}^{\perp}$, and $B_{i}=F_{\lambda} \oplus\left(B_{i} \cap F_{\lambda}^{\perp}\right), i=1,2$. Then

$$
\begin{aligned}
M: F_{\lambda} \oplus\left(B_{2} \cap F_{\lambda}^{\perp}\right) & \rightarrow\left(B_{0} \cap F_{\lambda}\right) \oplus\left(B_{0} \cap F_{\lambda}^{\perp}\right), \\
(x, y) & \rightarrow(u, v)=M(x, y)=\left(\psi_{x}, \psi_{y}\right),
\end{aligned}
$$

for some function $\psi$. Now consider the differential equation

$$
(x, y)_{t}=\nabla \psi
$$


Then

$$
\psi(x, y)_{t}=\psi_{x} u+\psi_{y} v=|\psi|^{2}>0
$$

if $(u, v) \neq(0,0)$. If $x=x(y, \lambda), u=0, v \neq 0$, we have $\psi_{y}(x(y, \lambda), \lambda) v(x(y, \lambda), \lambda)>0$. Thus if we define $\tilde{\psi}(y, \lambda)=\psi(x(y, \lambda), y)$, then when $x=x(y, \lambda), u=0, \phi=v$, and

$$
\begin{aligned}
\widetilde{\psi}(y, \lambda)_{s} & =\psi_{x} x_{y} \phi+\psi_{y} \phi \\
& =u x_{y} \phi+\psi_{y} v \\
& =\psi_{y} v>0
\end{aligned}
$$

if $v \neq 0$. Hence $\tau$ is the desired Lyapunov function.

Lemma 2.3. For $i=1,2, P\left(d \phi_{\left(0, \lambda_{i}\right)}\right) \approx P_{\lambda_{i}}$, where the symbol $\approx$ denotes isomorphism.

Proof. As before, we have

$$
\begin{aligned}
M:\left(F^{\perp} \cap B_{2}\right) \oplus F & \rightarrow\left(F^{\perp} \cap B_{0}\right) \oplus F \\
(x, y, \lambda) & \rightarrow(u, v)=M(x, y, \lambda),
\end{aligned}
$$

where $\lambda=\lambda_{1}$ or $\lambda_{2}$. Since $\phi(y)=v(x(y), y)$ (we are dropping the dependence on $\lambda$, for convenience), $d \phi=v_{x} x_{y}+v_{y}$. Also $u(x(y), y)=0$, so that $u_{x} x_{y}+u_{y}=0$. Hence in $\mathscr{U} \times \mathscr{N}$, we may write $x_{y}=-u_{x}^{-1} u_{y}$. Now since

$$
d M=\left(\begin{array}{ll}
u_{x} & u_{y} \\
v_{x} & v_{y}
\end{array}\right)
$$

the eigenvalue equation $d M(\beta, \alpha)^{t}=\mu(\beta, \alpha)^{t}$, gives the two equations

$$
\begin{aligned}
& \left(u_{x}-\mu\right) \beta+u_{y} \alpha=0 \\
& v_{x} \beta+\left(v_{y}-\mu\right) \alpha=0 .
\end{aligned}
$$

The eigenvalue equation for $d \phi$ reads

$$
0=(d \phi-\sigma) \gamma=\left(-v_{x} u_{x}^{-1} u_{y}+v_{y}-\sigma\right) \gamma .
$$

Now let $v \in P_{\lambda} \subset F$; then $v=(0, \alpha)^{t}$ and $d M v=\mu v$ gives $u_{y} \alpha=0$ and $\left(v_{y}-\mu\right) \alpha=0$. Thus from (2.5) with $\sigma=\mu$ and $\gamma=\alpha$, we get

$$
(d \phi-\mu) \alpha=\left(-v_{x} u_{x}^{-1} u_{y}+v_{y}-\mu\right) \alpha=0,
$$

so that $\alpha \in P\left(d \phi_{(0, \lambda)}\right)$. Next, we note that the map

$$
\begin{aligned}
& h: P_{\lambda} \rightarrow P\left(d \phi_{(0, \lambda)}\right) \\
& (0, \alpha)^{t} \rightarrow \alpha
\end{aligned}
$$

is clearly injective; thus $\operatorname{dim} P\left(d \phi_{(0, \lambda)}\right) \geqq \operatorname{dim} P_{\lambda}$. To show that $h$ is an isomorphism, it suffices to prove that

$$
\operatorname{dim} P_{\lambda} \geqq \operatorname{dim} P\left(d \phi_{(0, \lambda)}\right) .
$$


To do this, let $\alpha \in P\left(d \phi_{(0, \lambda)}\right)$, and define the vector $L(\alpha)$ by

$$
L(\alpha)=\left(\begin{array}{c}
-u_{x}^{-1} u_{y} \alpha \\
\alpha
\end{array}\right)
$$

Then $\alpha=\sum_{i=1}^{k} \alpha_{i}$, where $d \phi_{(0, \lambda)} \alpha_{i}=\mu_{i} \alpha_{i}$, each $\mu_{i} \geqq 0$, and $\alpha_{i} \perp \alpha_{j}$ if $i \neq j$. Hence

$$
\begin{aligned}
\left\langle d M_{\lambda} L(\alpha), L(\alpha)\right\rangle & =\left\langle\sum_{i} d M_{\lambda}\left(-u_{x}^{-1} v_{y} \alpha_{i}, \alpha_{i}\right)^{t}, \sum_{i}\left(-u_{x}^{-1} v_{y} \alpha_{i}, \alpha_{i}\right)^{t}\right\rangle \\
& =\left\langle\sum_{i}\left(0, \mu_{i} \alpha_{i}\right)^{t}, \sum_{i}\left(-u_{x}^{-1} v_{y} \alpha_{i}, \alpha_{i}\right)\right\rangle \\
& =\sum_{i, j}\left\langle\left(0, \mu_{i} \alpha_{i}\right)^{t},\left(-u_{x}^{-1} v_{y} \alpha_{j}, \alpha_{j}\right)^{t}\right\rangle \\
& =\sum_{i, j} \mu_{i} \alpha_{i} \cdot \alpha_{j}=\sum_{i} \mu_{i}\left|\alpha_{i}\right|^{2} \geqq 0 .
\end{aligned}
$$

Now write (c.f. Definition (2.1)), $B_{2}=P_{\lambda} \oplus N_{\lambda}$, and let $\pi$ denote the projection $\pi: P_{\lambda} \oplus N_{\lambda} \rightarrow P_{\lambda}$. Consider the map $\pi \circ L: P\left(d \phi_{(0, \lambda)}\right) \rightarrow P_{\lambda}$. We claim that this map is injective. Indeed, if $\pi \circ L(\alpha)=0$, then $L(\alpha) \in N_{\lambda}$, so if $L(\alpha) \neq 0$,

$$
\left\langle d M_{\lambda} L(\alpha), L(\alpha)\right\rangle<0
$$

which contradicts (2.8). Thus $\pi \circ L$ is injective and hence (2.7) holds. This completes the proof of the lemma.

End of proof of Theorem 2.1. First we remark that solutions of $M=0$ are in $1-1$ correspondence with the solutions of $\phi=0$. From Lemma 2.3 and hypothesis ii), $\operatorname{dim} P\left(d \phi_{\left(0, \lambda_{1}\right)}\right) \neq \operatorname{dim} P\left(d \phi_{\left(0, \lambda_{2}\right)}\right)$. Moreover, $d \phi_{\left(0, \lambda_{i}\right)}$ is non-singular since if for some $\alpha \neq 0 d \phi_{\left(0, \lambda_{i}\right)} \alpha=0$, then from (2.6), $\left(-v_{x} u_{x}^{-1} u_{y}+v_{y}\right) \alpha=0$. Hence $d M_{\lambda_{i}} L(\alpha)=(0,0)^{t}$, and this contradicts hypothesis (i). Now consider the finitedimensional flow $y_{s}=\phi(y, \lambda)$. The Conley indices $h\left(0, \lambda_{i}\right)$ of the isolated invariant set $\left(0, \lambda_{i}\right), i=1,2$, can be easily computed in this case; namely $h\left(0, \lambda_{i}\right) \approx \sum^{s_{i}}$ where $\sum^{s_{i}}$ is the pointed sphere of dimension $s_{i}=\operatorname{dimension} P_{\lambda_{i}}$; (see, e.g. [S]), and one finds $h\left(0, \lambda_{1}\right) \neq h\left(0, \lambda_{2}\right)$. From Conley's Continuation Theorem [C, S], if $\mathscr{V}$ is any neighborhood of 0 , then 0 cannot be the maximal invariant set in $\mathscr{V}$ for each $\lambda \in I$. Thus there exists $\lambda(\mathscr{V}) \in I$ such that 0 is not the maximal invariant set in $\mathscr{V}$ for the equation $y_{s}=\phi(y(s), \lambda(\mathscr{V}))$. It follows that the maximal invariant set in $\mathscr{V}$ for this equation contains a point $y \neq 0$. Since Lemma 2.2 implies that this equation is gradient-like, it follows that the $\alpha$ - and $\omega$-limit sets of $y$ are distinct rest points. Thus there must exist another rest point in $\mathscr{V}$ different from 0 . Now let the neighborhoods $\mathscr{V}$ shrink to 0 . As the corresponding $\lambda(\mathscr{V})$ 's have a convergent subsequence $\lambda\left(\mathscr{V}_{i}\right) \rightarrow \lambda_{0} \in I$, we see that $\left(0, \lambda_{0}\right)$ is a bifurcation point. Moreover, $\lambda_{1}<\lambda_{0}<\lambda_{2}$ since $d \phi_{\left(0, \lambda_{i}\right)}$ is non-singular, for $i=1,2$. It follows from what we have discussed above, that $\left(0, \lambda_{0}\right)$ is a bifurcation point for the equation $M(u, \lambda)=0$. This completes the proof of Theorem 2.1 . 


\section{§ 3. Equivariant Conley index}

In the standard form of the Conley Index Theory, a finite-dimensional generalization of the Morse index is constructed, and the theory is then extended to infinite dimensions via the notion of index pairs; see [C, S]. One can also consider the case when there exists a group $G$ of symmetries acting on the space $X$, and the flow (or semiflow) $\psi_{t}$, is equivariant in the sense that the group action commutes with the flow:

$$
\psi_{t}(g x)=g \psi_{t}(x) \quad \forall x \in X, \quad g \in G, \quad t \in \mathbb{R}\left(\text { or } \mathbb{R}_{+}\right) .
$$

In this section we shall assume that we are in this situation, and we shall show how to define an equivariant Conley index for any $G$-invariant isolated invariant set. Following Pacella, $[\mathrm{P}]$, we shall show that all the constructions of index pairs, isolating neighborhoods, the continuation theorem, etc., can be done using $G$-invariant neighborhoods.

The Conley index $h(I)$ of a non-degenerate critical point $I$ in finite dimensions, is given by $h(I)=\Sigma^{k}$, where $\Sigma^{k}$ denotes a pointed $k$-sphere, and $k$ is the number of positive eigenvalues of the linearized equations about $I$. More generally, if $V$ is the peigenspace of $I, h(I)$ is the homotopy type of the pointed space $D(V) / S(V)$, where $D(V)$ is the unit ball in $V$ and $S(V)$ the unit sphere in $V$. If we ignore the group action, then since $V$ is a vector space of dimension $k, D(V) / S(V) \approx \Sigma^{k}$. However, when there is a group acting on the space, we can put more structure on the Conley index; namely it becomes a pointed $G$-space. We write this index as $h_{G}(I)$. The advantage of using the equivariant Conley index is that it is possible to have $h\left(I_{1}\right)=h\left(I_{2}\right)$ but $h_{G}\left(I_{1}\right) \neq h_{G}\left(I_{2}\right)$, or $h\left(I, \lambda_{1}\right)=h\left(I, \lambda_{2}\right)$, but $h_{G}\left(I, \lambda_{1}\right) \neq h_{G}\left(I, \lambda_{2}\right)$. Therefore under such circumstances using the $h_{G}$ index it is possible to prove that bifurcation occurs while no such statement is possible from the ordinary Conley index. Moreover, just as in the non-equivariant case, at a non-degenerate critical point $I$ we may identify the Conley index with $D(V) / S(V)$, (where as above, $V=$ peigenspace of $I$ ), and it may happen that at distinct parameter values $\lambda_{1} \neq \lambda_{2}$, we have $\operatorname{dim} V_{1}=\operatorname{dim} V_{2}$, and thus $h\left(I, \lambda_{1}\right)=h\left(I, \lambda_{2}\right)$. But by a result of Lee and Wasserman, [LW], if $G=O(n)$ and $V_{1} \neq V_{2}$ as representations of $O(n)$, then $h_{O(n)}\left(I, \lambda_{1}\right) \neq h_{O(n)}\left(I, \lambda_{2}\right)$. It is thus quite useful to extend the notion of the Conley index to the equivariant case, and we shall now outline an "equivariant" Conley Index Theory.

Let $X$ be a metric space, let $G$ be a compact Lie group and let $\psi$ : $(\mathbb{R} \times G) \times X \rightarrow X$ be a $G$-flow on $X$; i.e., for all $t \in \mathbb{R}, g_{1}, g_{2} \in G$, and $x \in X$, we have

and

$$
\psi(0, e, x)=x, \quad(e=\text { id. element in } G),
$$

$$
\psi\left(t_{1}, g_{1}, \psi\left(t_{2}, g_{2}, x\right)\right)=\psi\left(t_{1}+t_{2}, g_{1} g_{2}, x\right) .
$$

Thus if $g=e, \psi(t, e, x) \equiv \psi_{t}(x)$ defines a flow on $X$. If $t=0, \psi$ induces an action of $G$ on $X$ by $\psi(0, g, x)=g x$. If $\pi$ is the canonical map

$$
\pi: X \rightarrow X / G,
$$


where $X / G$ is the orbit space of $X$ with respect to $G$, then if $\left(N_{1}, N_{0}\right)$ is an index pair in $X / G$, (c.f. [P]), then $\left(\pi^{-1}\left(N_{1}\right), \pi^{-1}\left(N_{0}\right)\right.$ ) is an index pair in $X$. If $I$ is a $G$-invariant set in $X$ (i.e., $g I \subset I \forall g \in G$ ), and $I$ is also an isolated invariant set for the flow $\psi_{t}$, we define $h_{G}(I)$, the equivariant Conley index of $I$ to be the equivariant homotopy type of the pointed space $\pi^{-1}\left(N_{1}\right) / \pi^{-1}\left(N_{0}\right)$ where $\left(N_{1}, N_{0}\right)$ is a index pair for $\pi(I)$. (We remark that Pacella, [P] has shown that there is an induced flow on the orbit space $X / G$, and if $I / G$ is an invariant set for the flow on $X / G$ then if $\left(N_{1}, N_{0}\right)$ is any index pair for $I / G$, $\left(\pi^{-1}\left(N_{1}\right), \pi^{-1}\left(N_{0}\right)\right)$ is a $G$-invariant index pair for $\left.I.\right)$

Now let $\left(N_{1}, N_{0}\right)$ and $\left(\tilde{N}_{1}, \tilde{N}_{0}\right)$ be $G$-invariant index pairs for $I$. Then there exist equivariant maps, (i.e., maps which commute with the $G$-action),

$$
\begin{aligned}
& f:\left(N_{1} / N_{0}, N_{0}\right) \rightarrow\left(\tilde{N}_{1} / \tilde{N}_{0}, \tilde{N}_{0}\right) \\
& h:\left(\tilde{N}_{1} / \tilde{N}_{0}, \tilde{N}_{0}\right) \rightarrow\left(N_{1} / N_{0}, N_{0}\right)
\end{aligned}
$$

such that both $h \circ f$ and $f \circ h$ are equivariantly homotopic to the identity map. That is, the pointed spaces $\left(N_{1} / N_{0}, N_{0}\right)$ and $\left(\widetilde{N}_{1} / \tilde{N}_{0}, \widetilde{N}_{0}\right)$ are of the same equivariant homotopy type. We thus can unambiguously define the $G$-invariant Conley index of $I, h_{G}(I)$, to be that equivariant homotopy type. Note that if $G=\{\mathrm{id}\}$, this definition agrees with the usual definition of the Conley index. The definition given here is somewhat richer since as the relevant spaces admit a group action, we can distinguish indices which have the same homotopy type as pointed topological spaces, but which are not of the same homotopy type as pointed $G$-spaces. We observe that the reason there exist maps $f$ and $h$ as defined above follows just as in [C, S]. All maps constructed there are easily seen to be $G$ equivariant if $G$ operates on the space and the index pairs $\left(N_{1}, N_{0}\right)$ and $\left(\tilde{N}_{1}, \tilde{N}_{0}\right)$ are $G$-invariant. In fact, all other things such as the fact that $h_{G}(I)$ is independent of the isolating neighborhood containing $I$, the existence of index pairs, Morse decompositions, and the Continuation Theorem all go through mutatis mutandis in the equivariant case. We remark that the advantage of defining the equivariant Conley index as a pointed space is that it is a "primary" definition. We can compose this index with any equivariant algebraic invariant to derive any other proposed definition of an equivariant Conley index; c.f. [P] for other definitions.

Finally we shall explain how the above ideas are used to extend the results of Sect. 2 in order to deduce an "equivariant" of Theorem 2.1. That is, we shall now that if the peigenspaces differ as $G$-spaces, then bifurcation must occur.

Thus let $B_{0}$ and $B_{2}$ be $G$-invariant Banach spaces, $H$ a $G$-invariant Hilbert space, $B_{2} \subset B_{0} \subset H$, where the embeddings are all continuous, and let $A$ be an interval in $\mathbb{R}$. Let $M: B_{2} \times A \rightarrow B_{0}$ be a smooth $G$-invariant operator $(M(g u, \lambda)$ $\left.=g M(u, \lambda) \forall g \in G, u \in B_{2}, \lambda \in \Lambda\right)$, satisfying

$$
M(0, \lambda)=0, \quad \forall \lambda \in \Lambda
$$

Since $M$ is $G$-invariant, it follows that the peigenspaces $P_{\lambda}$ and the neigenspaces $N_{\lambda}$ (see Definition 2.1) are also $G$-invariant. We assume that there is an $\varepsilon>0$ such that for all $\lambda \in \Lambda$ the peigenspace of $d M_{\lambda}+\varepsilon I$ is finite-dimensional, for each $\lambda \in A$. 
Definition 3.1. If $d M_{\lambda}$ is non-singular, the reduced $G$ index of $0, h_{R}^{G}(0, \lambda)$ is defined to be the equivariant homotopy type of the pointed $G$-space:

$$
h_{R}^{G}(0, \lambda)=D\left(P_{\lambda}\right) / S\left(P_{\lambda}\right),
$$

where $D\left(P_{\lambda}\right)$ denotes the unit ball in $P_{\lambda}$ and $S\left(P_{\lambda}\right)$ denotes the unit sphere in $P_{\lambda}$.

Note that in the finite-dimensional case, this definition coincides with our equivariant formulation of the Conley index given above.

Theorem 3.1. With $M$ defined as above and satisfying (3.1), assume that $M$ is a gradient for each $\lambda \in A$. Suppose that there exist $\lambda_{1}<\lambda_{2}$ in $A$ satisfying

(i) $d M_{\lambda_{i}}$ is non-singular for $i=1,2$,

(ii) $h_{R}^{G}\left(0, \lambda_{1}\right) \neq h_{R}^{G}\left(0, \lambda_{2}\right)$.

Then there exists a $\lambda_{0}, \lambda_{1}<\lambda_{0}<\lambda_{2}$ such that $\left(0, \lambda_{0}\right)$ is a bifurcation point for $M(u, \lambda)=0$.

Notice that if $G=\{\mathrm{id}\}$, the trivial group, then this theorem reduces to Theorem 2.1.

The connection of hypothesis (ii) with the peigenspaces is made via the following theorem of Lee and Wasserman [LW].

Theorem 3.2. If $G=O(n)$ and $V$ and $W$ are representations of $O(n)$, then the pointed $O(n)$-spaces $D(V) / S(V)$, and $D(W) / S(W)$ are equivariantly homotopy equivalent iff $V \approx W$ as $O(n)$ representations.

Theorem 3.2 is valid for other groups besides $O(n)$. Thus, we call a group $G$ "nice" if whenever $V$ and $W$ are two vector spaces satisfying $D(V) / S(V)$ $\sim_{G}(D(W) / S(W)$ (same equivariant homotopy type as $G$-spaces), then $V \approx W$ as representations of $G$. Then if $G_{0}$ denotes the component of $G$ containing the identity, $G$ is nice if, for example, $G / G_{0}=\mathbb{Z}_{2} \times \ldots \times \mathbb{Z}_{2}$, (so $G=O(n)$ is nice), or $G / G_{0}=\mathbb{Z}_{3} \times \ldots \times \mathbb{Z}_{3}$, or $G / G_{0}=\mathbb{Z}_{4}$, or $G$ is connected, (so $G=S O(n)$ and $G=U(n)$ are nice); see [LW]. On the other hand there are groups $G$ and distinct representations $V$ and $W$ with $D(V) / S(V)$ equivariantly homeomorphic to $D(W) /$ $S(W)$; see [CS].

In view of Theorem 3.2, we see that Theorem 3.1 is equivalent to the following theorem, (since $\left.h_{R}^{O(n)}\left(0, \lambda_{i}\right)=D\left(P_{\lambda_{i}}\right) / S\left(P_{\lambda_{i}}\right), i=1,2\right)$ :

Theorem 3.3. Assume that the hypotheses of Theorem 3.1 hold but with (ii) replaced by

(ii)' $P_{\lambda_{1}}$ is not isomorphic to $P_{\lambda_{2}}$ as a representation of $O(n)$. Then the conclusion of Theorem 3.1 is valid.

The proof of Theorem 3.1 is virtually the same as that of Theorem 2.1. In the statement of Lemma 2.3, $P\left(d \phi_{\left(0, \lambda_{i}\right)}\right) \approx P_{\lambda_{i}}$, must be understood now as meaning that the two spaces are isomorphic as $G$-spaces. The map $h$ in the proof of Lemma 2.3 is also equivariant (i.e., $h(g x)=g h(x) \forall g \in G$ ), since the spaces $P_{\lambda}$ and $P_{\lambda}^{\perp}$ are invariant under the group action. The global, (in $\lambda$ ), LyapunovSchmidt reduction given in Lemma 2.3 can easily be done equivariantly, and Theorem 3.3, which states that if the peigenspaces differ as $G$-spaces, then bifurcation occurs, is a strengthened version of Theorem 2.1. 
Proof of Theorem 3.2. If $V \approx W$ as $O(n)$ representations, then there is an equivariant linear isomorphism $L: V \rightarrow W$ and so $D(V) / S(V)$ and $D(W) / S(W)$ are equivariantly homotopy equivalent. To prove the converse we first recall that a representation is determined by its character; i.e., two representations are the same if they have equal characters. Now by [LW, Th. 2.21], if $V$ and $W$ are two representations of $G$, and $D(V) / S(V)$ is equivariantly homotopy to $D(W) / S(W)$, and $o\left(G / G_{0}\right)=p^{k}$ where $p$ is a prime number, and $G_{0}$ is the component of the identity in $G$, then the derivative, $D[\chi(V)-\chi(V)] \equiv 0$. Thus the difference in characters, $\chi(V)-\chi(W)$ must be constant on components of $G$. Now if $G=O(n)$, we shall now $\chi(V)-\chi(W) \equiv 0$. First note that $G_{0}=S O(n)$ and $o\left(G / G_{0}\right)=2$. Next, as $D(V) / S(V)$ is homeomorphic to the sphere $S^{\operatorname{dim} V}$, it follows that $\operatorname{dim} V=\operatorname{dim} W$. Also as $\chi(V)(e)=\operatorname{dim} V(e=$ identity in $G)$, we see that $\chi(V)=\chi(W)$ on the identity component of $G$. Now if $G=O(n)$, we can find an element $g \in O(n)$ such that $g^{2}=e$; for example, let $g=\operatorname{diag}(-1,1, \ldots, 1)$. Then a calculation gives $\chi(V) g$ $=2 \times \operatorname{dim} V^{G}-\operatorname{dim} V$, where $V^{G}$ is the fixed point set of $V$; i.e., those elements in $V$ fixed by all $g \in G$ (see (6.1) below). Thus $\chi(V) g-\chi(W) g=2\left(\operatorname{dim} V^{G}\right.$ $\left.-\operatorname{dim} W^{G}\right)=0$ from [LW, Theorem 2.7]. Hence $\chi(V)=\chi(W)$ so that $V=W$, and our theorem is proved.

\section{$\S$ 4. Semilinear elliptic equations - A summary of the linear results}

In this section we begin to discuss the application of our results to the problem of symmetry-breaking for solutions of (1.1), (1.2). In an earlier paper, [ $\left[\mathrm{SW}_{5}\right]$, we showed that for a fairly broad class of nonlinear $C^{1}$-functions $f$, "infinitesimal" symmetry-breaking must occur in the sense that for a fixed nodal class, there exist infinitely-many degenerate radial solutions of (1.1), on distinct balls, the kernels of whose linearized operators contain asymmetric elements. We shall use this result to show that actual symmetry-breaking occurs.

The symmetry-breaking problem presents certain difficulties which do not occur in the bifurcation problems usually encountered. One such difficulty is that we do not bifurcate off of a "trivial" branch (i.e., $u \equiv 0$ ) of solutions, and if one transforms the problem so that we do bifurcate off of a trivial branch, we lose a certain variational structure so that the basic bifurcation theorems, (e.g. [B, Kr, R]), cannot be applied. Another difficulty is that one cannot directly apply the Crandall-Rabinowitz theorem. There are two reasons for this: namely, if $n>1$, we never bifurcate from a "simple eigenvalue", and secondly, the "transversality" hypothesis is quite difficult to verify. However, there is a way to finesse the first difficulty, (by considering $O(n-1)$-invariant functions; see $\left[\mathrm{SW}_{2}\right]$ ), but the verification of the transversality condition remains a difficult open problem. (However see $\left[\mathrm{SW}_{3}\right]$, where the transversality condition is verified for positive solutions of the Dirichlet problem.)

For these reasons, we have found it necessary to develop a different approach to the symmetry-breaking problem. Our method is based on the Conley index and can be described as follows. We show that in any given nodal class, the radial solutions $u(\cdot, \lambda)$ can be parametrized by a real parameter $\lambda>0, \lambda>\bar{\lambda}$. We then show that there is an increasing sequence $\lambda_{i} \rightarrow \infty$ for which the peigen- 
spaces $P_{\lambda_{i}}$ satisfy the hypotheses of Theorem 3.3, so that bifurcation must occur. We then give an additional argument in order to show that radial bifurcation can be avoided so that the symmetry actually breaks.

The verifications of the hypotheses of Theorem 3.3 concerning the peigenspaces $P_{\lambda_{2}}$ follows easily from $\left[\mathrm{SW}_{5}\right]$. It is here where we must place additional hypotheses on $f$ in order to rule out certain highly degenerate situations. For example, if we consider radial solutions lying in a $k>1$ nodal class, we require $f$ to be analytic.

We remark too that in using the Conley index, we must pay a certain price, this being that we cannot assert the existence of a connected branch of bifurcating solutions - in fact, we do not know the precise structure of the bifurcating set; (see [Po] for an interesting algebraic example). On the other hand, there are some interesting advantages to using the Conley index technique. One is that by employing an equivariant from of the Conley index, as discussed in the last section, we are able to avoid certain "cancellation of indices", which may occur in the non-equivariant theory. That is, we can prove that bifurcation occurs even if the usual Conley index has not changed, provided that there is a change in the representation. This enables us to prove that symmetrybreaking occurs under less restrictive hypotheses. Another advantage of this technique is that we can obtain a richer bifurcating set in the sense that we can show that distinct solutions come out having distinct symmetries. This aspect is discussed in Sect. 6. Finally, the use of the Conley index enables us to avoid requiring that the eigenvalues be "simple", or "odd-dimensional", or that they "cross transversally" at bifurcation points, as required in the theorems of Crandall-Rabinowitz, [S], or Krasneselskii, [Kr], Böhme, [B], and others.

In the remainder of this section we shall formulate the problem, and recall the main results in $\left[\mathrm{SW}_{5}\right]$. In the next section we shall consider in detail the verification of the hypotheses of Theorem 3.3.

We consider the equations (1.1), (1.2) where we assume that the function $f$ satisfies the following hypotheses:

There exist points $b<0<\gamma$ such that:

(i) $f(\gamma)=0, f^{\prime}(\gamma)<0$

(ii) $F(\gamma)>F(u)$ if $b<u<\gamma$ (here $F^{\prime}=f$ and $F(0)=0$ )

(iii) $F(b)=F(\gamma)$

(iv) if $f(b)=0$, then $f^{\prime}<0$

(v) $u f(u)+2(F(\gamma)-F(u))>0$ if $b<u<\gamma$.

These conditions have been discussed in $\left[\mathrm{SW}_{5}\right]$; we remark that conditions (ii) and (v) are implied by the condition $u f(u)>0$ if $u \in(b, \gamma) \backslash\{0\}$. We shall have more to say below, about the import these conditions have to our symmetrybreaking problem.

Now radial solutions of (1.1), (1.2) satisfy the equation

$$
u^{\prime \prime}(r)+\frac{n-1}{r} u^{\prime}(r)+f(u(r))=0, \quad 0<r<R, \quad r=|x|,
$$

together with the boundary conditions

$$
u^{\prime}(0)=0=\alpha u(R)-\beta u^{\prime}(R) .
$$


The solution of the initial-value problem for (4.2) with $u(0)=p>0, u^{\prime}(0)=0$, will be denoted by $u(r, p)$, and $p$ will be considered as a parameter. We define angles $\theta_{0}$ and $\theta(r, p)$ by

$$
\begin{aligned}
\theta_{0} & =\operatorname{Tan}^{-1}(\alpha / \beta), \quad-\pi / 2 \leqq \theta_{0}<\pi / 2, \quad \text { and } \\
\theta(r, p) & =\operatorname{Tan}^{-1}\left(u^{\prime}(r, p) / u(r, p)\right) .
\end{aligned}
$$

If $k$ is a given non-negative integer, and $f(p)>0$, we define the function $T_{k}(p)$ by

$$
\theta\left(T_{k}(p), p\right)=\theta_{0}-k \pi
$$

It was proved in [SW $\mathrm{SW}_{5}$, Prop. 2.2], that $T_{k}(p)$ is well-defined for $p$ near $\gamma$. Note that $T_{k}(p)$ plays the role of $R$, and so $R$ now varies with $p$. Since $k$ will be fixed, in what follows, we write $T_{k}(p)=T(p)$. A solution of (4.2), (4.3), and (4.6) will be said to belong to the " $k^{\text {th }}$ nodal class" of $f$. With these preliminaries out of the way, we can now summarize the main results of $\left[\mathrm{SW}_{5}\right]$.

The principal result in $\left[\mathrm{SW}_{5}\right]$ states that there are infinitely-many points $p_{j}, 0<p_{j}<\gamma$ for which the corresponding solutions $u\left(\cdot, p_{j}\right)$ of $(4.2),(4.3)$, (4.6) are "degenerate", in the sense that the kernel of the corresponding linearized operator is singular and contains asymmetric elements. More precisely suppose that the dimension $n$, and the nodal class $k$ are given. Then there is an integer $\bar{N}$ having the following property: For every integer $N \geqq \bar{N}$, there are $k$-solutions $u\left(\cdot, p_{j}^{N}\right), j=1,2, \ldots, k, \lim _{N \rightarrow \infty} p_{j}^{N}=\gamma$, of $(4.2),(4.3),(4.6)$ such that the linearized problem

$$
\begin{aligned}
\left.\Delta w(x)+f^{\prime}\left(u(x), p_{j}^{N}\right)\right) w(x)=0, & & |x|<T\left(p_{j}^{N}\right), \\
\alpha w(x)-\beta d w(x) / d n=0, & & |x|=T\left(p_{j}^{N}\right)
\end{aligned}
$$

admits solutions of the form $a(r) \Phi_{N}(\theta)$, where $a \equiv 0$ and $\Phi_{N}$ lies in the $N^{\text {th }}$ eigenspace of the Laplacian on the $(n-1)$-sphere $S^{n-1}$. Furthermore, the peigenspace at $u\left(\cdot, p_{k}^{N}\right)$ contains exactly $j$ copies of $E_{N}$ and less than $j$ copies of $E_{N+1}$ (if $j>0$ ). From the results in $\left[\mathrm{SW}_{5}\right]$, it is easy to show that we may choose $p_{j}^{N+1}>P_{j}^{N}$ if $N \geqq \bar{N}$. Thus we can say that "the symmetry-breaks infinitesimally" for all sufficiently high modes if $k>0$. Moreover, if hypotheses (4.1), (ii) and (v) are replaced by the stronger hypotheses and

(ii) $u f(u)>0$ if $b<u<0$, or $0<u<\gamma$,

(v) $f_{\mathrm{a}}^{\prime}(0)>0$,

then the integer $\bar{N}$ is universal, i.e., it is independent of $f$. The main purpose of this section is to strengthen these results by showing that the symmetry breaks, in the sense that actual bifurcation to asymmetric solutions occurs on infinitely-many degenerate radial solutions.

In order to study the bifurcation problem, it is necessary to work in a fixed space of functions; i.e.; we wish to remove the dependence of $R=T(p)$, on $p$. We accomplish this by a simple scaling device, namely, if $u(x)$ solves (1.1), (1.2) on the $n$-ball $|x|<R$, then the function $z(y)=u(R y)$ solves the equation

$$
\Delta z(y)+R^{2} f(z(y))=0, \quad|y|<1,
$$


together with the boundary conditions

$$
\alpha w(y)-R \beta d w(y) / d n=0, \quad|y|=1 .
$$

Notice that $R$ appears explicitly in the boundary conditions (4.9) only if both $\alpha$ and $\beta$ are non-zero; i.e., Dirichlet or Neumann boundary conditions do not explicitly involve the parameter $R$ in (4.9). The explicit dependence of $R$ in the boundary conditions causes certain technical difficulties, in that we cannot consider pairs $(u, R)$ lying in a product space of the form $B \times \mathbb{R}$. Thus we cannot apply bifurcation theory in the usual sense. To get around this, we show in the appendix, that the set

$$
\left\{(u, R) \in C_{\hat{\partial}}^{2}\left(D^{n}\right) \times \mathbb{R}_{+}: \alpha u(x)-\beta R d u(x) / d n=0,|x|=1\right\}
$$

forms a vector bundle over $\mathbb{R}_{+}$, whose fiber at $R$ equals $p^{-1}(R)$, where $p$ is the projection on the first factor; i.e., for any $R_{0}$ and sufficiently small $\varepsilon$, we show that $p^{-1}\left[\left(R_{0}-\varepsilon, R_{0}+\varepsilon\right)\right]$ is a product. This local product structure is sufficient to apply the usual bifurcation techniques, as we also show in the appendix.

\section{§ 5. Symmetry-breaking bifurcations}

We shall show here that the results of Sect. 2 and 3 apply to the symmetrybreaking problem for (1.1), (1.2). Thus we shall that for $N \geqq \bar{N}$ (c.f. the discussion preceding (4.7)), that we can find an increasing sequence of numbers $\lambda_{N} \rightarrow \infty$ for which the peigenspaces $P_{\lambda_{N}}$ satisfy the hypotheses of Theorem 3.3; i.e. $d M_{\lambda_{N}}$ is non-singular and $P_{\lambda_{N}}$ and $P_{\lambda_{N+1}}$ differ as representations of $O(n)$. Thus there must be infinitely-many bifurcation points, $u\left(\cdot, \bar{\lambda}_{N}\right), \lambda_{N}<\bar{\lambda}_{N}<\lambda_{N+1}$. As we have mentioned before, it does not follow that the symmetry breaks on these solutions; indeed, $u\left(\cdot, \bar{\lambda}_{N}\right)$ could be a "radial" bifurcation point. In other words, there can bifurcate out of $u\left(\cdot, \bar{\lambda}_{N}\right)$ a branch of radial solutions. We shall, however, prove that this doesn't occur if $\lambda_{N}$ is sufficiently large, so that the symmetry must break on such solutions.

The actual verification of the hypotheses of Theorem 3.3 is made difficult since one must avoid certain degenerate situations whereby it is conceivable that zero lies in the spectrum of the linearized operators for an entire open interval of $\lambda$ 's. For example, if $u^{\prime}$ changes sign, it is possible to have distinct elements $a_{N}(r) \Phi_{N}(\theta), a_{M}(r) \Phi_{m}(\theta), N \neq M$, in the kernel of the linearized operator about a given radial solution $u(\cdot, \lambda)$; see $\left[\mathrm{SW}_{2}\right.$, Theorem 3.4]. This situation is depicted in Fig. 1. Thus as $\lambda$ varies, different elements $a_{N} \Phi_{N}$ could enter and leave the kernel, so as to render degenerate an entire continuum of solutions $u(\cdot, \lambda)$. However, we shall show that this problem can be avoided if the function $f$ is assumed to be analytic. This leads us to consider separately the cases $u^{\prime}(r, \lambda)<0,0 \leqq r \leqq 1$, and the general case where $u^{\prime}$ is allowed to change sign; only in the latter case need we assume $f$ to be analytic. In order to carry out the above program, we find it technically more convenient in computing peigenspaces, to consider the family of radial solutions parametrized 


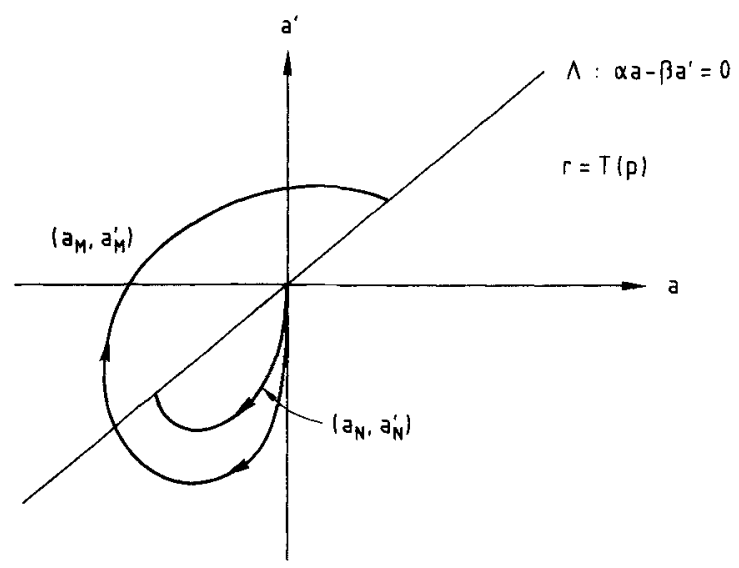

Fig. 1

by $p$, rather than by $\lambda$; as we show in the appendix, this does not affect the computation of indices.

Under the hypotheses (4.1) on $f$, if $k$ is a fixed positive integer, then there is a $s_{0}<\gamma$ such that $\left[s_{0}, \gamma\right) \subset \operatorname{dom}(T) \equiv \operatorname{dom}\left(T_{k}\right)$; (see $\left.\left[\mathrm{SW}_{5}\right]\right)$. Also, as was shown in $\left[\mathrm{SW}_{4}\right]$, there is an $s_{1}$ with $s_{0}<s_{1}<\gamma$ such that $T_{k}^{\prime}(p)>0$ if $s_{1} \leqq p<\gamma$. For such $p$, the linearized operator $L^{p} w=\Delta w+f^{\prime}(u(\cdot, p)) w$, defined on functions $w$ satisfying the boundary conditions $(1.2)(R=T(p))$, has a finite number of positive eigenvalues each of finite multiplicity. If $\psi$ is an eigenfunction of the Laplacian on $S^{n-1}$ with eigenvalue $\lambda_{N}=-N(N+n-2)$ and $\phi$ is a radial function satisfying the given boundary conditions on $|x|=T(p)$ then $w=\phi(r) \psi$ satisfies the boundary conditions and $L^{p} w=\left(L_{N}^{p} \phi\right) \psi$ where

$$
L_{N}^{p} \phi=\phi^{\prime \prime}+\frac{n-1}{r} \phi^{\prime}+\left[f^{\prime}(u(\cdot, p))+\frac{\lambda_{N}}{r^{2}}\right] \phi .
$$

Thus, $L_{N}^{p}$ has no positive eigenvalue for large $N$. Also, if $L_{N+1}^{p}$ has $j$ positive eigenvalues, then $L_{N}^{p}$ has (at least) $j$ positive eigenvalues. On the other hand, for $p$ close to $\gamma, L_{N}^{p}$ has $k$ (or $k+1$ ) positive eigenvalues of multiplicity $l_{N}$. Since the eigenvalues of $L_{N}^{p}$ are continuous in $p$, we can find $p_{N}^{j}<\gamma$, for $0 \leqq j \leqq k$, such that $L_{N}^{p_{N}}$ has exactly $j$ positive eigenvalues of multiplicity $l_{N}$, if $N$ is large. (The proofs of the above statements can all be found in $\left[\mathrm{SW}_{5}\right]$ ). We shall show below that we can choose the points $p_{N}^{j}$ such that each $L^{p_{N}}$ is non-singular. This will imply that the conditions of Theorem 3.3 are satisfied, so that we have bifurcation on some $u(\cdot, p), p$ between $p_{N}^{j}$ and $p_{N}^{j+1}$. Furthermore the bifurcation must be non-radial since $T^{\prime}(p)>0$ (see $\left[\mathrm{SW}_{2}\right]$ ); i.e., symmetry-breaking occurs.

We begin by considering the case of monotone radial solutions.

Theorem 5.1. Assume that $f$ satisfies hypotheses (4.1). Suppose that we consider monotone radial solutions of (4.2), (4.3) with $0 \leqq \theta_{0}<\pi / 2$ and thus $k=1$. Then there exists an increasing sequence of numbers $\lambda_{m} \rightarrow \infty$ for which the corresponding 
radial solutions $u_{\lambda_{m}}(\cdot)$ are symmetry-breaking bifurcation points. Equivalently, there exists a sequence $\bar{p}_{m} \nearrow \gamma$ for which the radial solutions $u\left(\cdot, p_{m}\right)$ are symmetrybreaking bifurcation points. The kernels of the associated linearized operators have dimensions at least

$$
l_{m}=\left(\begin{array}{c}
m+n+2 \\
n
\end{array}\right)\left(\frac{n+2 m-2}{n+m-2}\right)
$$

Proof. We recall from $\left[\mathrm{SW}_{5}\right]$, the definitions of the operators $L_{N}^{p}: \Psi_{p} \rightarrow C(0, T(p))$, where

namely,

$$
\Psi_{p}=\left\{\phi \in C^{2}(0, T(p)): \phi(0)=\alpha \phi(T(p))-\beta \phi^{\prime}(T(p))=0\right\} ;
$$

$$
L_{N}^{p} \phi=\phi^{\prime \prime}+\frac{n-1}{r} \phi^{\prime}+\left(\frac{\lambda_{N}}{r^{2}}+f^{\prime}((r, p))\right) \phi
$$

We endow $\Psi_{p}$ with the following inner product:

$$
\langle\phi, \psi\rangle=\int_{0}^{T(p)} \phi(r) \psi(r) r^{n-1} d r
$$

Then with $\bar{N}$ defined as above, and $i \in \mathbb{Z}_{+}$satisfying $i \geqq \bar{N}$, let ${ }^{1}$

$$
p_{i}=\sup \left\{p: \sigma\left(L_{i}^{p}\right)<0, s_{1}<p<\gamma\right\}
$$

here $\sigma\left(L_{i}^{p}\right)$ denotes the sup of the spectrum of the operator $L_{i}^{p}$. At this point we need a lemma.

Lemma 5.2. If $p<p_{i}$, then $\sigma\left(L_{j}^{p}\right)<0$ for $j \geqq i$.

Proof. For $\phi \in \Psi_{p}$,

$$
\begin{aligned}
\left\langle L_{j}^{p} \phi, \phi\right\rangle & =\int_{0}^{T(p)}\left[\phi\left(r^{n-1} \phi^{\prime}\right)^{\prime}+\left(f^{\prime}(u(r, p))+\frac{\lambda_{j}}{r^{2}}\right) \phi^{2} r^{n-1}\right] d r \\
& \leqq \int_{0}^{T(p)}\left[\phi\left(r^{n-1} \phi^{\prime}\right)^{\prime}+\left(f^{\prime}(u(r, p))+\frac{\lambda_{i}}{r^{2}}\right) \phi^{2} r^{n-1}\right] d r \\
& =\left\langle L_{i}^{p} \phi, \phi\right\rangle<0,
\end{aligned}
$$

from which the result follows.

Corollary 5.3. If $u(\cdot, p)$ is a monotone solution of (4.2), (4.3), then $p_{i+1}>p_{i}$ for all $i \geqq \bar{N}$.

\footnotetext{
${ }^{1}$ It was shown in [ $\mathrm{SW}_{5}$, Propositions 4.3 and 4.5], that if $i \geqq \bar{N} \sigma\left(L_{i}^{p}\right)<0$ for certain $p$ 's, $s_{1}<p<\gamma$
} 
Proof. Our lemma implies $p_{i+1} \geqq p_{i}$, and since $u^{\prime}(r, p) \leqq 0$, it follows from [SW Theorem 3.4] that $p_{i+1}>p_{i}$ if $i \geqq \bar{N}$.

We can now complete the proof of Theorem 5.1. Thus fix $i \geqq \bar{N}$, and consider solutions $a_{N}$ of the initial-value problem for the equation $L_{N}^{p_{i}} a=0$, for varying $N$. The orbit segments $\left(a_{i}(r), a_{i}^{\prime}(r)\right),\left(a_{j}(r), a_{j}^{\prime}(r)\right), j<i$, and $\left(a_{l}(r), a_{l}^{\prime}(r)\right), l>i$, at $r$ $=T\left(p_{i}\right)$, are depicted in Fig. 2 . That is, if $j<i$, the orbit $\left(a_{j}(r), a_{j}^{\prime}(r)\right)$ has crossed the boundary line $A=\alpha a-\beta \alpha^{\prime}=0$, and for $l>i$, the orbit $\left(a_{l}(r), a_{l}^{\prime}(r)\right)$ has not crossed this line. In fact each operator $I_{N}^{p_{i}}$ has exactly one positive eigenvalue if $i<N$ and has negative spectrum if $i>N$. These facts are consequences of Proposition 3.16 in $\left[\mathrm{SW}_{3}\right]$.

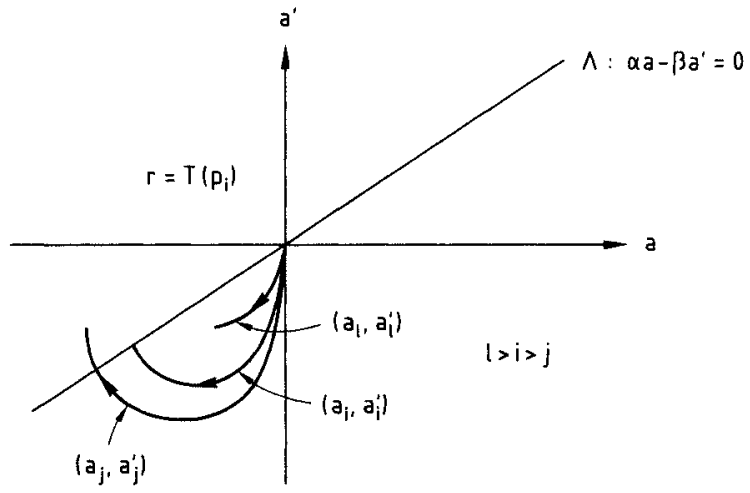

Fig. 2

Thus the operator $\Delta+f^{\prime}\left(u\left(\cdot, p_{i}\right)\right.$ ), (defined on $C^{2}$ functions $\phi$ on $|x|<T(p)$, satisfying the given boundary conditions), has exactly $(i-1)$-positive eigenvalues; namely

$$
\left\{\mu^{j}\left(p_{i}\right): i \leqq j \leqq i-1\right\},
$$

where

$$
\mu^{j}(p)=\sup _{\phi}\left\langle L_{j}^{p} \phi, \phi\right\rangle .
$$

Let $\lambda(p)$ denote the number of positive eigenvalues of the operator $\Delta+f^{\prime}(u(\cdot, p))$, again on functions in $C^{2}(|x| \leqq T(p))$ which satisfy the boundary conditions.

Now $\mu^{i-1}(p)$ must become positive for some $p<p_{i}$; namely, since the orbit $\left(a_{i-1}(r), a_{i-1}^{\prime}(r)\right)$ at $r=T\left(p_{i}\right)$ has crossed $\Lambda$, (see Fig. 2$), \mu^{i-1}(p)$ cannot stay $\leqq 0$ for all $p<p_{i}$. Thus (see Fig. 3), there exists points $q_{i}, p_{i-1}<q_{i}<p_{i}$ such that $\mu^{i-1}\left(q_{i}\right)>0, \lambda\left(q_{i}\right)=i-1$, and

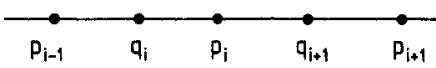

Fig. 3 
this is true for every $i \geqq \bar{N}$. Now we claim that zero is not in the spectrum of the operator $\Delta+f^{\prime}(u(\cdot, p))$, satisfying the given boundary conditions. To see this, note that $L_{-1}^{q_{i}}$ has no zero eigenvalue (since $\mu^{i-1}\left(q_{i}\right)>0$ ), and if $j \geqq i, L_{i}^{q_{i}}$ has negative spectrum, while if $j<i-1$, the orbit $\left(a_{j}(r), a_{j}^{\prime}(r)\right)$ has crossed the boundary line $A$ when $r=T\left(q_{i}\right)$. Thus our claim holds so that the solutions $u\left(\cdot, q_{i}\right)$ are non-degenerate for each $i \geqq \bar{N}$. It follows that the peigenspaces $P_{i}$ $=P\left(u\left(\cdot, q_{i}\right)\right)$ satisfy

$$
P_{i} \neq P_{i+1}, \quad \text { as } O(n) \text { representations for } i \geqq \bar{N} \text {. }
$$

Thus Theorem 3.1 together with the result in the appendix, imply that there are points $\bar{q}_{i}, q_{i}<\vec{q}_{i}<q_{i+1}$ such that $u\left(\cdot, \bar{q}_{i}\right)$ is a bifurcation point. Now as in $\left[\mathrm{SW}_{3}\right]$, we can show that $q_{i} \rightarrow \gamma$ as $i \rightarrow \infty$. Moreover, as $T^{\prime}(p)>0, s_{1}<p<\gamma$, it follows that there is an integer $i_{0} \in \mathbb{Z}_{+}$for which

$$
T^{\prime}\left(\bar{q}_{i}\right)>0, \quad i \geqq i_{0} .
$$

Next, as shown in [ $\left.\mathrm{SW}_{2}\right]$, condition (5.5) together with hypothesis (4.1) (v) implies that radial bifurcation cannot occur. It follows then, that the symmetry breaks on $u\left(\cdot, \bar{q}_{i}\right)$, if $i \geqq i_{0}$. This completes the proof of the theorem.

We now turn to the case where the radial solutions are not monotone; i.e., the case where $u^{\prime}(\cdot, p)$ changes sign. As we have mentioned above, we find it necessary to assume here that $f$ is analytic.

Theorem 5.4. Let $f$ be an analytic function satisfying conditions (4.1), and consider radial solutions of (4.2), (4.3), (4.6), (which are not necessarily monotone). Then there exists an increasing sequence of points, $\bar{p}_{m} \rightarrow \gamma$, for which the corresponding radial solutions, $u\left(\cdot, \bar{p}_{m}\right)$, are symmetry-breaking bifurcation points. Equivalently, there is a sequence $\lambda_{m} \rightarrow \infty$ for which the corresponding radial solution $u\left(\cdot, \lambda_{m}\right)$ are symmetry-breaking bifurcation points.

Proof. Since $f$ is analytic, it follows that any (smooth) radial solution $u(r, p)$ of (4.2) is analytic as a function of both $r$ and $p$. (The proof of this statement for $p$ is the same as in the $C^{k}$-case; see the appendix in $\left[\mathrm{SW}_{1}\right]$.) Now denote by $a(r, p)$ the solution of

$$
\begin{gathered}
a^{\prime \prime}+\frac{n-1}{r} a^{\prime}+\left(f^{\prime}(u(r, p))+\frac{\lambda_{i}}{r^{2}}\right) a=0, \quad 0<r<T(p), \\
a(0)=0=\alpha a(T(p), p)-\beta a^{\prime}(T(p), p) .
\end{gathered}
$$

Since (5.6) has a regular singular point at $r=0$, the well-known result of Frobenius shows that this equation has a one-dimensional space of solutions analytic in $r$ and $p$ at $r=0$, and hence for all $r \geqq 0$. Let $a_{N}$ be the unique analytic solution satisfying $a_{N}(r) / r^{N} \rightarrow 1$ as $r \rightarrow 0$ (c.f. Lemma 3.6 of $\left[\mathrm{SW}_{5}\right]$ ). 
Now define the function $\phi_{N}(r, p)$ by

$$
\phi_{N}(r, p)=\operatorname{Tan}^{-1}\left(a_{N}^{\prime}(r, p) / a_{N}(r, p)\right),
$$

and note that $\phi_{N}(T(p), p)$ is an analytic function if $T(p)$ is analytic, where $T(p)$ is defined in (4.6). Moreover, using the implicit function theorem, we see that $T(p)$ is analytic provided that $\theta^{\prime}(T(p), p) \neq 0$.

Lemma 5.5. Let $f$ be analytic; then for $p$ near $\gamma, \phi_{N}(T(p), p)$ is an analytic function. Proof. We have, from (4.5), and (4.6)

$$
\left(u^{2}+v^{2}\right) \theta^{\prime}(T(p), p)=-\frac{n-1}{T(p)} u v-u f(u)-v^{2},
$$

where $u$ and $v$ are evaluated at $(T(p), p)$. Note that if $\alpha=0$ or $\beta=0,(5.9)$ implies $\theta^{\prime}(T(p), p) \neq 0$ if $p$ is near $\gamma$; this follows easily from hypothesis (4.1) (v). We may thus assume $\alpha \neq 0$ in what follows. Now along the level curve $H(u, v) \equiv v^{2} / 2$ $+F(u)=H(\gamma, 0),\left(v=u^{\prime}\right)$, we have $v^{2}=2(F(\gamma)-f(u))$, so that $(4.2)(v)$ implies that $u f(u)+v^{2}>0$ along this level curve. We claim that this level curve meets the boundary line $A: \alpha u-\beta v=0$ transversally. Indeed, if we compute the derivative $d H / d \mu$ of $H$ in the direction tangent to $A$, we find $d H / d \mu=(\beta, \alpha) \cdot \nabla H=$ $\beta f(u)+\alpha v$. Thus on the line $\beta / \alpha=u / v$, we find that $(v / \alpha) d H / d \mu=u f(u)+v^{2}>0$ along the level curve; this proves the claim. Let $Q=(\bar{u}, \bar{v})$ denote the point of intersection of $A$ with $H(u, v)=H(\gamma, 0)$; c.f. Fig. 4. Now using the same sort of arguments as Proposition 2.2 of $\left[\mathrm{SW}_{5}\right]$, we find that for $p$ near $\gamma$, the orbit $(u(r, p), v(r, p))$ meets $A$ at a point $\left(u_{p}, v_{p}\right)$ near $Q$. If $J(u, v)=u f(u)+v^{2}$, then $J(\bar{u}, \bar{v})>0$ so for $p$ near $\gamma, J\left(u_{p}, v_{p}\right) \geqq J(\bar{u}, \bar{v}) / 2$. Choosing $p$ so

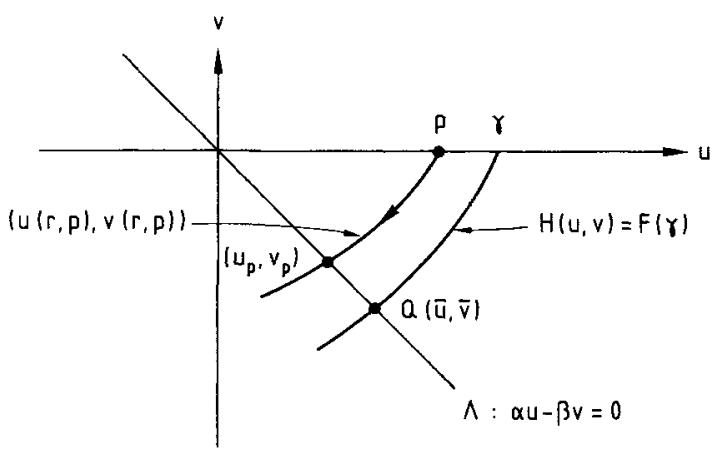

Fig. 4

close to $\gamma$ so that $|(n-1) u v / T(p)|<J(u, v) / 4$, we see that for such $p, \theta^{\prime}(T(p), p)<0$, and this completes the proof of the lemma.

We can now complete the proof of Theorem 5.4. To this end, note first that from our lemma, there is an interval $I=\left[p_{0}, \gamma\right], p_{0}>0$, such that $T(p)$ 
is analytic on $I$; thus $\phi_{N}(p) \equiv \phi_{N}(T(p), p)$ is analytic on $I$ for all $N$. Choose $M_{0} \in \mathbb{Z}_{+}, M_{0} \geqq \bar{N}$ such that

$$
\phi_{M_{0}}\left(p_{0}\right)>\theta_{0}
$$

(c.f. Fig. $5 \mathrm{a}$ ); this can be achieved using Lemma 3.6 of $\left[\mathrm{SW}_{5}\right]$. Thus in some interval $U_{0}$ about $p_{0}$,
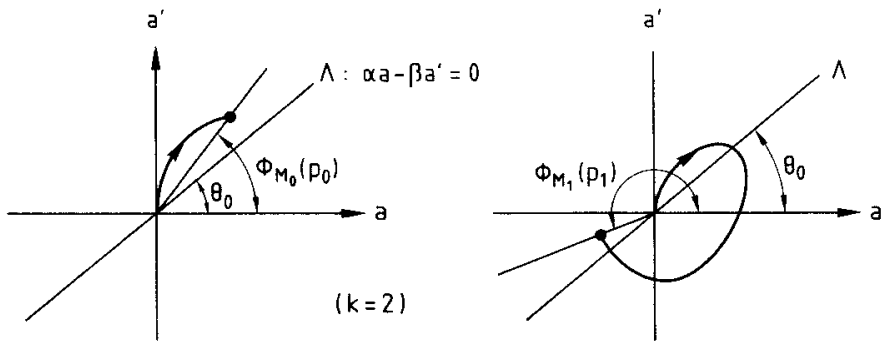

Fig. 5

$$
\max _{p \in U_{0}} \lambda(p) \leqq(k+1)\left(1+l_{1}+l_{2}+\ldots l_{M_{0}-1}\right) \equiv L
$$

where $l_{M}$ is defined in (5.1), and as above, $\lambda(p)$ denotes the number of positive eigenvalues of the operator $A+f^{\prime}(u(\cdot, p))$ defined on functions in $C^{2}(|x| \leqq T(p))$ satisfying the given boundary conditions.

Choose $M_{1} \in \mathbb{Z}_{+}$such that $M_{1}>M_{0}$ and

$$
(k+1) l_{M_{1}}>L
$$

Using Proposition 3.15 in $\left[\mathrm{SW}_{5}\right]$, we can find a point $p_{1}, p_{0}<p_{1}<\gamma$ such that (c.f. Fig. 5 b),

$$
\phi_{M_{1}}\left(p_{1}\right)<\theta_{0}-k \pi
$$

It follows that in some interval $U_{1}$ about $p_{1}$,

$$
\phi_{M_{1}}(p)<\theta_{0}-k \pi, \quad p \in U_{1}
$$

Note that $U_{0} \cap U_{1}=\phi$ since (5.12) and (5.14) imply that

$$
\lambda(p) \geqq(k+1) l_{M_{1}}>L, \quad \text { if } p \in U_{1} .
$$

Now $\phi_{N}(p)$ is an analytic function of $p$ for every integer $N$. Thus for each $N, \phi_{N}(p)=\theta_{0}$ for at most a finite number of $p$ 's. We can thus find points $q_{0} \in U_{0}$, and $q_{1} \in U_{1}$ such that $\phi_{M_{0}}\left(q_{0}\right) \neq \theta_{0}, \phi_{M_{0}}\left(q_{1}\right) \neq \theta_{0}$; i.e., the radial functions $u\left(\cdot, q_{0}\right)$, and $u\left(\cdot, q_{1}\right)$ are non-degenerate. Consequently (5.11) and (5.15) imply that the peigenspaces $P\left(u\left(\cdot, q_{0}\right)\right)$ and $P\left(u\left(\cdot, q_{1}\right)\right)$ satisfy

$$
\operatorname{dim} P\left(u\left(\cdot, q_{0}\right)\right)=r, \quad \text { and } \quad \operatorname{dim} P\left(u\left(\cdot, q_{1}\right)\right)=s, \quad r<L<s,
$$


$r=\lambda\left(q_{0}\right)$, and $s=\lambda\left(q_{1}\right)$. That is, the dimensions of the peigenspaces, at these non-degenerate solutions are different.

Next choose $M_{2} \in \mathbb{R}_{+}$such that $(k+1) l_{M_{2}}>\lambda\left(q_{1}\right)$, and $M_{2}>M_{1}$. As before, we can find a point $p_{2}, q_{1}<p_{2}<\gamma$ such that $\theta_{M_{2}}\left(p_{2}\right)<\theta_{0}-k \pi$. We can choose a neighborhood $U_{2}$ of $p_{2}$ for which $\theta_{M_{2}}(q)<\theta_{0}-k \pi$ if $q \in U_{2}$. Also, for such $q$, we have $\lambda(q) \geqq(k+1) l_{M_{2}}>\lambda\left(q_{1}\right)$. Again using the fact that $\theta_{M_{2}}(p)$ is an analytic function of $p$, we can conclude that there is a point $q_{2} \in U_{2}$ with $\lambda\left(q_{2}\right)>\lambda\left(q_{1}\right)$; i.e.,

$$
\operatorname{dim} P\left(u\left(\cdot, q_{2}\right)\right)=\lambda\left(q_{2}\right) \neq \lambda\left(q_{1}\right)=\operatorname{dim} P\left(u\left(\cdot, q_{1}\right)\right)
$$

Continuing this procedure yields a monotone sequence $\left\{q_{k}\right\}$ having the property that

$$
\operatorname{dim} P\left(u\left(\cdot, q_{k}\right)\right) \neq \operatorname{dim} P\left(u\left(\cdot, q_{k+1}\right)\right), \quad k=1,2, \ldots,
$$

and each $u\left(\cdot, q_{k}\right)$ is non-degenerate. Another application of Theorem 3.1 yields the existence of bifurcation points $\left\{u\left(\cdot, \bar{p}_{k}\right): k=1,2, \ldots\right\}$, and as in the proof of the last theorem, the symmetry breaks on each such solution if $k$ is sufficiently large.

Using Theorem 3.1, we can do a finer analysis. For example, we can find points $q_{N}^{j}, 1 \leqq j \leqq k$ in $\left(s_{1}, \gamma\right)$ such that $q_{N}^{j}<q_{N+1}^{j}$ for each $j$, and $u\left(\cdot, q_{N}^{j}\right)$ is nondegenerate, and the peigenspace $P\left(u\left(\cdot, q_{N}^{j}\right)\right)$ has $j$-copies of $E_{N-1}$ but $<j$ copies of $E_{N}$. Thus Theorem 3.1 implies that there is a point $\bar{q}_{N}^{j} \in\left(q_{N}^{j}, q_{N+1}^{j}\right)$ such that $u\left(\cdot, \bar{q}_{N}^{j}\right)$ is a bifurcation point. We thus get a $k$-fold infinite family of bifurcation points. To see this, set, for $1 \leqq j \leqq k$,

$$
p_{N}^{j}=\inf \left\{p \in\left(s_{1}, \gamma\right): \phi_{N}(p)=\theta_{0}-j \pi\right\} .
$$

Then from the results in $\left[\mathrm{SW}_{5}\right], \phi_{N-1}\left(p_{N}^{j}\right)<\theta_{0}-j \pi$ so $\phi_{N-1}(p)<\theta_{0}-j \pi$ for $p<p_{N}^{j}$ and $p$ close to $p_{N}^{j}$. Choose $q_{N}^{j}$ to be such a point where $u\left(\cdot, q_{N}^{j}\right)$ is non-degenerate. Then at $u\left(\cdot, q_{N}^{j}\right)$ the peigenspace has $j$-copies of $E_{N-1}$ and $<j$ copies of $E_{N}$.

\section{§ 6. Distinguishing solutions by their symmetry subgroups}

In this section we shall show how to obtain different asymmetric solutions of (1.1), (1.2) at each bifurcation point. (Of course, we assume that $f$ satisfies hypotheses (4.1) and that $f$ is analytic if $k>1$.) How this will be achieved can best be understood by reviewing the non-equivariant case. Thus, from [ $\mathrm{SW}_{5}$, Theorem 3.1], we know that for every $N \in \mathbb{Z}_{+}$, there is a point $s_{N}, 0<s_{N}<\gamma$, $s_{N}<s_{N+1}, s_{N} \rightarrow \gamma$, such that if $s_{N}<p<\gamma$, then the spectrum of $L_{N}^{p}$ (c.f. (5.3)) contains $k$-positive elements. If $l_{j}$ is the dimension of $E_{j}$, the $j^{\text {th }}$ eigenspace of the Laplacian on $S^{n-1}$, then (c.f. (5.1))

$$
l_{j}=\left(\begin{array}{c}
j+n-2 \\
j
\end{array}\right)\left(\frac{2 j+n-2}{j+n-2}\right) .
$$


Thus if $A_{p}$ denotes the peigenspace of the radial solution $u(\cdot, p)$, then $\operatorname{dim} A_{p}$ $\geqq k \sum_{j=0}^{N} l_{j}$. It follows that as $p \rightarrow \gamma, \operatorname{dim} \Lambda_{p} \rightarrow \infty$. These changes in dimensions are what enabled us to prove the existence of infinitely-many symmetry-breaking solutions. We want to use similar techniques, now taking the group action into account, in order to show that solutions bifurcate out having different symmetry groups.

Let $H$ be a subgroup of $O(n)$, and consider the set

$$
\Lambda_{p}^{H}=\left\{w \in \Lambda_{p}: w(h x)=w(x) \quad \forall h \in H, \forall x \in D_{T(p)}^{n}\right\} .
$$

$\Lambda_{p}^{H}$ is called the fixed-point set of $H$ in the peigenspace $\Lambda_{p}$ of the radial solution $u(\cdot, p)$. By Theorem 3.1, if $\Lambda_{p_{1}} \neq \Lambda_{p_{2}}$ and $d M_{\left[u\left(\cdot, p_{i}\right), p_{i}\right]}$ are non-singular, $i=1,2$ there is a point $p$ between $p_{1}$ and $p_{2}$ for which $u(\cdot, p)$ is a bifurcation point. But the hypothesis $\Lambda_{p_{1}} \neq \Lambda_{p_{2}}$ does not necessarily imply $\Lambda_{p_{1}}^{H} \neq \Lambda_{p_{2}}^{H}$ (as representations of $N(H) / H$, where $N(H)$ denotes the normalizer of $H ; \Lambda_{p}^{H}$ is not necessarily invariant under $G$ but is invariant under $N(H)$, and in fact $N(H) / H$ acts on this space.) Moreover, even if $\Lambda_{p_{1}}^{H} \neq \Lambda_{p_{2}}^{H}$, this does not imply bifurcation on some $u(\cdot, p)$ (for $p$ between $p_{1}$ and $p_{2}$ ), since $N(H) / H$ may have more than four components, and we cannot apply the results in Sect. 3 ; in particular the results in [LW] do not apply. On the other hand, if $\operatorname{dim} \Lambda_{p_{1}}^{H} \neq \operatorname{dim} \Lambda_{p_{2}}^{H}$, then there is a $p$ between $p_{1}$ and $p_{2}$ such that $u(\cdot, p)$ is a bifurcation point. (Proof: In a manner similar to what we have done in Sect. 2, consider $M: B_{2}^{H} \times A \rightarrow B_{0}^{H}$, perform the same Lyapunov-Schmidt reduction, and conclude as before, that on the finite dimensional space, the Conley indices are distinct at $p_{1}$ and $p_{2}$ so bifurcation occurs.) The following abstract general theorem will allow us to assert the existence of many bifurcating solutions at each bifurcation point.

Theorem 6.1. Suppose that the hypotheses of Theorem 3.1 hold and assume that $(0, \lambda)$ are the only invariant solutions of $M(u, \lambda)=0$ for each $\lambda \in\left[\lambda_{1}, \lambda_{2}\right]$, and that $\operatorname{ker}\left[d M_{\lambda}\right]$ contains no invariant elements. Let $H$ and $K$ be subgroups of $G$ satisfying

$$
\operatorname{dim} P_{\lambda_{1}}^{H} \neq \operatorname{dim} P_{\lambda_{2}}^{H}, \quad \text { and } \quad \operatorname{dim} P_{\lambda_{1}}^{K} \neq \operatorname{dim} P_{\lambda_{2}}^{K},
$$

HK, the group generated by $H$ and $K$ satisfies $H K=G$.

Then there exist $\lambda_{H}$ and $\lambda_{K}$ in $\left(\lambda_{1}, \lambda_{2}\right)$ such that $\left(0, \lambda_{K}\right)$ (resp. $\left.\left(0, \lambda_{H}\right)\right)$ is a bifurcation point of solutions with symmetry (at least) $H$ (resp. $K$ ). The bifurcating solutions do not coincide.

Proof. The existence of bifurcation points $\left(0, \lambda_{H}\right)$ and $\left(0, \lambda_{K}\right)$ follows from (i) in view of our above remarks. Since $\operatorname{ker}\left(d M_{\lambda}\right)$ contains no invariant elements, no invariant bifurcation is possible (see [D, Remark on p. 288]). On the other hand, any solution fixed by both $H$ and $K$ must be fixed by $H K$ and thus must be invariant, because of (ii). It follows that the bifurcating solutions must be distinct.

We now apply this result to the problem (1.1), (1.2).

${ }^{2}$ More generally, $h^{N(H) / H}\left(0, \lambda_{1}\right) \neq h^{N(\boldsymbol{H}) / H}\left(0, \lambda_{2}\right)$ and $h^{N(K) / K}\left(0, \lambda_{1}\right) \neq h^{N(K) / K}\left(0, \lambda_{2}\right)$; see [LW] 
Theorem 6.2. Assume that $f$ satisfies hypotheses (4.1) and consider radial solutions of (4.2), (4.3), (4.6). If $k>1$, assume that $f$ is also analytic. Let $H$ and $K$ be subgroups of $O(n)$ satisfying condition (ii) of Theorem 6.1. Suppose that for $p$ near $\gamma$, the kernel of the linearized operator about $u(\cdot, p)$ contains no purely radial element. Assume too that at each degenerate solution both a $K$-invariant and an $H$ invariant element comes into the peigenspace. Then there exist $p_{n} \rightarrow \gamma$ such that $u\left(\cdot, p_{n}\right)$ is a bifurcation point. At each $u\left(\cdot, p_{n}\right)$ there bifurcates out solutions $\left\{u_{n, \alpha}^{H}\right\}$, and $\left\{u_{n, \beta}^{K}\right\}$ having symmetry groups at least $H$ and $K$ respectively, and these solutions are distinct; i.e., $u_{n, \alpha}^{H} \neq u_{n, \beta}^{K}$ for all $\alpha$ and $\beta$.

Proof. We have seen in Sect. 4 that the points where 0 lies in the spectrum of the linearized operators forms a discrete set. At these degenerate points the $H$ - and $K$-invariant peigenspaces change; hence Theorem 6.1 is applicable and the theorem is proved.

We shall now find subgroups of $O(n)$ for which the hypotheses of our theorem can be verified. We first need some notation. For $p, q \in \mathbb{R}, 1 \leqq p, q \leqq n$, let $O_{n}(p)$ be that subgroup of $O(n)$ consisting of matrices of the form

$$
\begin{array}{rr}
p & n-p \\
p\left\{\left[\begin{array}{rr}
* & 0 \\
0 & I
\end{array}\right],\right.
\end{array}
$$

and let $\widetilde{O}_{n}(q)$ denote the subgroup of $O(n)$ consisting of matrices of the form

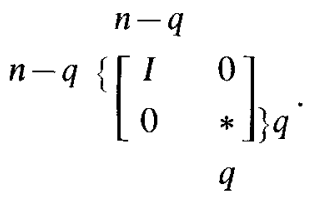

Lemma 6.3. Let $l \leqq p<q \leqq n$, and let $H=O_{n}(p) \times \widetilde{O}_{n}(n-p), K=O_{n}(q) \times \widetilde{O}_{n}(n-q)$. Then $K H=O(n)$.

Proof. We shall prove the stronger statement

$$
O_{n}(p+1) O_{n}(n-p)=O(n)
$$

then as the elements in $K$ are of the form

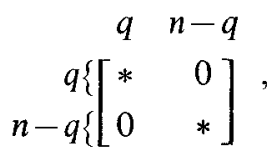

we see that $O_{n}(p+1) \subset K$. Since $O_{n}(n-p) \subset H,(6.5)$ implies that $K H \subset O(n)$.

Our proof of (6.3) will be by induction. To this end, we fix $n-p$, and proceed by induction on $n$. Let $L \in O(n)$, and set $L\left(e_{i}\right)=v_{i}, i=1,2, \ldots, n$, where $\left\{e_{i}\right\}$ denotes the standard unit vector basis in $\mathbb{R}^{n}$. We first show that there exist elements

$$
T_{1} \in O_{n}(p+1), \quad S_{1} \in \widetilde{O}_{n}(n-p) \quad \text { such that } S_{1}\left(T_{1} e_{1}\right)=v_{1} .
$$


To do this, let $v_{1}=\left(x_{1}, \ldots, x_{n}\right)$, and choose $T_{1} \in O_{n}(p+1)$ such that

$$
T e_{1}=\left(x_{1}, \ldots, x_{p}, t, 0, \ldots, 0\right) \quad \text { where } t=\left(\sum_{p+1}^{n} x_{i}^{2}\right)^{1 / 2} .
$$

This can be done since $O_{n}(p+1)$ acts transitively on $S^{p}$. Now if $t=0$ then (6.4) obviously holds. Thus suppose $t \neq 0$, and choose $S_{1} \in \widetilde{O}_{n}(n-p)$ such that

$$
S_{1}\left(e_{p+1}\right)=\frac{1}{t}\left(0, \ldots, 0, x_{p+1}, \ldots, x_{n}\right) .
$$

Then

so (6.4) holds.

$$
\begin{aligned}
S_{1} T_{1} e_{1} & =S_{1}\left(x_{1}, \ldots, x_{p}, t, 0, \ldots, 0\right) \\
& =\left(x_{1}, \ldots, x_{p}, x_{p+1}, \ldots, x_{n}\right)=v_{1},
\end{aligned}
$$

Now the orthogonal transformation $T_{1}^{-1} S_{1}^{-1} L$ fixes $e_{1}$ so $T_{1}^{-1} S_{1}^{-1} L \in \widetilde{O}_{n}(n-1)$. Thus by our induction hypothesis, $T_{1}^{-1} S_{1}^{-1} L \in \widetilde{O}_{n}(p) \widetilde{O}_{n}(n-p)$, where $\tilde{\widetilde{O}}_{n}(p)$ denotes the subset of $O(n)$ of consisting of matrices of the form

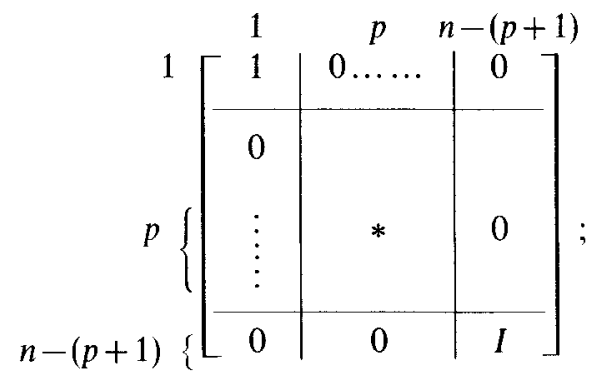

that is, $\widetilde{O}_{n}(p+1)$ operates on $e_{2}, \ldots, e_{P+1}$. Thus $T_{1}^{-1} S_{1}^{-1} L \in O_{n}(p+1) \widetilde{O}_{n}(n-p)$ so that $L \in O_{n}(p+1) \widetilde{O}_{n}(n-p)$, and this completes the proof.

Now in order to apply Theorem 6.2, we must verify (6.1) and (6.2) for the groups $H=O_{n}(p) \times \widetilde{O}_{n}(n-p)$ and $K=O_{n}(q) \times \widetilde{O}_{n}(n-q), 1 \leqq p<q \leqq n$. To do this, we need the notion of "isotropy group". Thus, if $u$ is a solution of (1.1), (1.2), the isotropy group at $u$, or the stability subgroup of $u, O(n)_{u}$ is defined by

$$
O(n)_{u}=\left\{g \in O(n): u(g x)=u(x), \forall x \in D_{R}^{n}\right\}
$$

Now it is sometimes possible to prove the existence of a solution $u_{1}$ whose isotropy group contains a given group $H_{1}$; i.e., $O(n)_{u_{1}} \supset H_{1}$. If $u_{2}$ is another solution and $O(n)_{u_{2}} \supset H_{2}$, then $u_{1}$ can be distinguished from $u_{2}$ if we know that $H_{1} H_{2}=O(n)$, and not both $u_{1}$ and $u_{2}$ are radial. In particular since we know that for $p$ near $\gamma$, radial bifurcation cannot occur $\left[\mathrm{SW}_{2,4}\right]$, then if for each $p, 1 \leqq p \leqq n$, we can prove the existence of a solution having isotropy sub- 
group containing $O(p) \times \widetilde{O}(n-p)$ then Lemma 5.2 shows that solutions with isotropy group $H_{1} \supset O\left(p_{1}\right) \times \widetilde{O}\left(n-p_{1}\right)$, and solutions with isotropy group $H_{2} \supset O\left(p_{2}\right) \times \tilde{O}\left(n-p_{2}\right),\left(0<p_{1}<p_{2} \leqq n\right)$, do not lie in the same orbit. Thus we can say that solutions have distinct orbits by considering their stability subgroups. In this way we can obtain many different bifurcating solutions via Theorem 6.1. [Note too that the groups $O(p) \times \widetilde{O}(n-p)$ and $O(n-p) \times \widetilde{O}(p)$ are conjugate in $O(n)$; namely if $u$ is a solution with symmetry group $O(p) \times \widetilde{O}(n-p)$, there is a $g \in O(n)$ such that $g u$ is a solution having symmetry group $O(n-p) \times \widetilde{O}(p)$. In order to rule out this trivial case, we shall assume $1 \leqq p \leqq n / 2$.]

The existence of bifurcating solutions having symmetry group $O(p) \times \widetilde{O}(n-p)$ is a consequence of the next two lemmas; they will imply that (6.1) is satisfied; we have already proved (6.2) in Lemma 6.3.

Lemma 6.4. Let $1 \leqq p \leqq n$, and set $H=O(p) \times \widetilde{O}(n-p)$. If $\mathbb{R}\left[x_{1}, \ldots, x_{n}\right]$ denotes the set of all polynomials in $n$ variables, with real coefficients, then

$$
\mathbb{R}\left[x_{1}, \ldots, x_{n}\right]^{H}=\mathbb{R}\left[r^{2}, \tilde{r}^{2}\right],
$$

where $\mathbb{R}\left[x_{1}, \ldots, x_{n}\right]^{H}$ denotes the set of elements in $\mathbb{R}\left[x_{1}, \ldots, x_{n}\right]$ fixed by $H$, $r^{2}=x_{1}^{2}+\ldots+x_{p}^{2}$, and $\tilde{r}^{2}=x_{p+1}^{2}+\ldots+x_{n}^{2}$.

Proof. Let $p \in \mathbb{R}\left[r^{2}, \tilde{r}^{2}\right]$. If $T \in O(p) \times \tilde{e}$, then $T$ preserves $r^{2}$ and doesn't affect $x_{p+1}, \ldots, x_{n}$, so $r^{2}$ and $\tilde{r}^{2}$ are unchanged by $T$. Similarly, if $S \in e \times \widetilde{O}(n-p), r^{2}$ and $\tilde{r}^{2}$ are likewise unchanged by $S$. It follows that $\mathbb{R}\left[r^{2}, \tilde{r}^{2}\right] \subseteq \mathbb{R}\left[x_{1}, \ldots, x_{n}\right]^{H}$. For the reverse inclusion, assume $p\left(x_{1}, \ldots, x_{n}\right) \in \mathbb{R}\left[x_{1}, \ldots, x_{n}\right]^{H}$. Now there are elements $T \in O(p), S \in \widetilde{O}(n-p)$ such that

$$
\begin{aligned}
T\left(x_{1}, \ldots, x_{p}\right) & =(t, 0, \ldots, 0), & t^{2}=r^{2} \\
S\left(x_{p+1}, \ldots, x_{n}\right) & =(s, 0, \ldots, 0), & s^{2}=\tilde{r}^{2} .
\end{aligned}
$$

Thus

$$
p\left(x_{1}, \ldots, x_{n}\right)=(T \times S) p\left(x_{1}, \ldots, x_{n}\right)=p(t, 0, \ldots, 0, s, 0, \ldots, 0) .
$$

Next, $\exists T^{\prime} \in O(p)$ such that $T^{\prime}\left(x_{1}, x_{2}, \ldots, x_{p}\right)=\left(-x_{1}, x_{2}, \ldots, x_{p}\right)$, and $\exists S^{\prime} \in \widetilde{O}(n-p)$ such that $S^{\prime}\left(x_{p+1}, \ldots, x_{n}\right)=\left(-x_{p+1}, x_{p+2}, \ldots, x_{n}\right)$. Thus

$$
\begin{aligned}
p\left(x_{1}, \ldots, x_{n}\right) & =\left(T^{\prime} T \times S\right) p\left(x_{1}, \ldots, x_{n}\right) \\
& =p\left(\left(T^{\prime} T \times S\right)\left(x_{1}, \ldots, x_{n}\right)\right) \\
& \left.=p\left(\left(T^{\prime} T\right)\left(x_{1}, \ldots, x_{p}\right)\right), S\left(x_{P+1}, \ldots, x_{n}\right)\right) \\
& =p(-t, 0, \ldots, 0, s, 0, \ldots, 0) .
\end{aligned}
$$

Similarly, $p\left(x_{1}, \ldots, x_{n}\right)=p(t, 0, \ldots, 0,-s, 0, \ldots, 0)$. Thus $p\left(x_{1}, \ldots, x_{n}\right)=q\left(t^{2}, s^{2}\right)$, where $q$ is a polynomial, so that $p\left(x_{1}, \ldots, x_{n}\right)=q\left(r^{2}, \tilde{r}^{2}\right) \in \mathbb{R}\left[r^{2}, \tilde{r}^{2}\right]$.

Lemma 6.5. Let $E_{k}$ denote the $k^{\text {th }}$ eigenspace of the Laplacian on $S^{n-1}$, and let $H=O(p) \times \widetilde{O}(n-p), 1 \leqq p<n$. Then $\operatorname{dim} E_{k}^{H}=1$, if $k$ is even. 
Proof. We identify $E_{k}$ with the space of homogeneous, harmonic polynomials of degree $k$ in $n$-variables. Let $P_{k}$ denote the homogeneous polynomials of degree $k$ in $n$-variables, and consider the following commutative diagram:

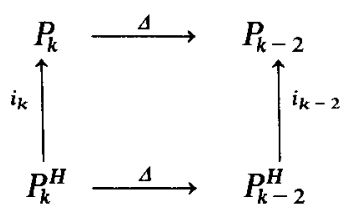

where $i_{k}$ and $i_{k-2}$ are inclusion maps. We shall show that $\left.\Delta\right|_{P_{k}^{H}}$ is surjective. Once having proved this, it follows that

$$
\operatorname{dim} E_{2 k}^{H}=\operatorname{dim} P_{2 k}^{H}-\operatorname{dim} P_{2(k-1)}^{H}=(k+1)-k=1,
$$

since by our last lemma, a basis for $P_{2 k}^{H}$ is given by the $(k+1)$-elements $r^{2 k}, \tilde{r}^{2} r^{2 k-2}, \ldots, \tilde{r}^{2 k}$.

Now it is well known that $\Delta: P_{k} \rightarrow P_{k-2}$ is surjective (c.f. $\left[\mathrm{SW}_{2}\right]$ ). Thus let $q \in P_{k-2}^{H}$; then there is a $p \in P_{k}$ such that $\Delta p=q$. If $\mu$ denotes (normalized) Haar measure on $H_{1}$ then as $h q=q$ for all $h \in H$, we have

$$
\int_{H} h q d \mu=\int_{H} q d \mu=q \int_{H} d \mu=q .
$$

It follows that

$$
q=\int_{\boldsymbol{H}} h \Delta p d \mu=\int_{\boldsymbol{H}} \Delta(h p) d \mu=\Delta\left[\int_{\boldsymbol{H}}(h p) d \mu\right] .
$$

But since $\bar{p}=\int_{H}(h p) d \mu$ is invariant under $H$, we see that $\bar{p} \in P_{k}^{H}$ and thus $\left.\Delta\right|_{P k^{K}}$ is surjective.

Alternatively, we take as a basis for $P_{2 k}^{H}$ the elements $r^{2 a} \tilde{r}^{2(k-a)}, a=0,1, \ldots, k$, (Lemma 6.4), and we compute:

$$
\begin{aligned}
\Delta\left(r^{2 a} \tilde{r}^{2(k-a)}\right)= & \left(\Delta r^{2 a}\right) \tilde{r}^{2(k-a)}+r^{2 a} \Delta\left(\tilde{r}^{2(k-a)}\right) \\
= & 2 a(p+2 a-2) r^{2(a-1)} \tilde{r}^{2(k-a)} \\
& +2(k-a)[(k-p)+2(k-a)-2] r^{2 a} \tilde{r}^{2(k-a-1)} .
\end{aligned}
$$

If $S$ is the subspace of $P_{2 K}^{H}$ generated by the set $\left\{r^{2 a} \tilde{r}^{2(k-a)}: 1 \leqq a \leqq k\right\}$, then we see that the matrix for the Laplacian defined on $S$ to $P_{2 k-2}^{H}$ is triangular in this basis, with all non-zero entries on the diagonal. Hence $\left.\Delta\right|_{S}$ is injective and thus surjective. It follows that $\Delta$ restricted to $P_{2 k}^{H}$ is surjective.

Notice that this lemma implies that (6.1) holds for the groups $O(q) \times \widetilde{O}(n-q)$, $1 \leqq q<n$, since as $p \rightarrow \gamma, p>p_{N}$, implies that $\operatorname{dim}\left(\Lambda_{p}^{H}\right) \geqq k N$. We thus have the following theorem.

Theorem 6.6. Let $f$ satisfy hypotheses (4.1), and assume that $f$ is analytic if $k \geqq 2$. Let $H_{p}=O(p) \times \widetilde{O}(n-p), 1 \leqq p \leqq n / 2$. Then there are points $q_{j} \rightarrow \gamma,\left(q_{j}<\gamma\right)$, such 
that at the radial solution $u\left(\cdot, q_{j}\right)$ the symmetry breaks, and there bifurcate out distinct solutions having symmetry groups (at least) $H_{p}, 1 \leqq p \leqq n / 2$, and $O(n-1)$. (The statement regarding $O(n-1)$ was proved in $\left[\mathrm{SW}_{2}\right]$.)

It is still an open problem to describe the entire bifurcation set; i.e., what else bifurcates out?

\section{Appendix}

The quantity $p=u(0)$, which has been used to parametrize the family of radial solutions $u(\cdot, p)$ of $(1.1),(1.2)$ is unsuitable as a bifurcation parameter. This is due to the fact that in bifurcation theory, one must work on a fixed product space $B \times A$ where $B$ is a Banach space of functions, and $A$ denotes the parameter space. But in our case, choosing $p$ as parameter forces the radii $R$ of the balls to vary with $p$, (i.e., $R=T(p)$ ), so that the parameter appears in the function space. The obvious way to overcome this difficulty is to perform a change of scale, taking $|x| \leqq R$ to the unit ball, and indeed, for homogeneous Dirichlet or Neumann boundary conditions, $(\alpha \beta=0)$, this technique works and we recover the desired product structure. However, if $\alpha b \neq 0$, it turns out that the parameter enters the boundary conditions and we again do not have a product structure. It is the purpose of this section to show that our problem nevertheless is equivalent to one on a fixed ball with fixed boundary conditions. In fact, our development will be such that all of the usual bifurcation theorems, e.g. the CrandallRabinowitz Theorem, [S], as well as the bifurcation results obtainable via the Conley Index Theory, (as discussed in $\S 2$ and $\S 3$ ), are applicable. Our technique is to prove that the relevant space forms a vector bundle over $\mathbb{R}$, and is thus locally a product. We then show that this local product structure suffices for doing bifurcation theory.

Consider the system (1.1), (1.2), which we rewrite for convenience as

$$
\begin{aligned}
\Delta U(z)+f(U(z))=0, & z \in D_{R}^{n}, \\
\alpha U(z)-\beta d U(z) / d n=0, & z \in \partial D_{R}^{n} .
\end{aligned}
$$

Here, as we have seen in $\S 4, R=T(p)$ on radial solutions $U(\cdot, p)$. Thus, if we change scale by writing

$$
x=z / \lambda, \quad u(x)=U(\lambda x), \quad \lambda=R,
$$

then the problem $\left(A_{1}\right),\left(A_{2}\right)$ goes over into the problem

$$
\begin{aligned}
& \Delta u(x)+\lambda^{2} f(u(x))=0, \quad x \in D^{n} \equiv D_{1}^{n} \\
& \alpha u(x)-\beta \lambda d u(x) / d n=0, \quad x \in \partial D^{n},
\end{aligned}
$$

where now $\lambda$ is considered as the parameter, and $\lambda \neq 0$. Notice however that the boundary conditions $\left(\mathrm{A}_{5}\right)$ vary with $\lambda$, unless $\alpha=0$ or $\beta=0$; i.e., unless 
we are considering the Dirichlet or Neumann problem. Hence, if $\alpha \beta=0$, the problem $\left(\mathrm{A}_{4}\right),\left(\mathrm{A}_{5}\right)$ can be considered as one defined on a (product) space

$$
\begin{array}{ll}
\left\{(u, \lambda) \in C^{2}\left(D^{n}\right) \times \mathbb{R}_{+}: u(x)=0 \text { if } x \in \partial D^{n}\right\}, & \text { if } \beta=0, \\
\left\{(u, \lambda) \in C^{2}\left(D^{n}\right) \times \mathbb{R}_{+}: d u(x) / d n=0 \text { if } x \in D^{n}\right\}, & \text { if } \alpha=0 .
\end{array}
$$

In either of these cases we have the usual framework for bifurcation theorems. But if $\alpha \beta \neq 0$, (the only case we consider, in what follows), the space of functions again depends on the parameter $\lambda$, and we still do not have a product structure. We shall show, however, that the space of functions forms a "vector bundle" over $\mathbb{R}$ (c.f. [L]) and is thus locally a product space of the desired form. We shall then transport the differential equation to this product space, so that the transformed system fits into the standard framework of bifurcation theory. We can then check that the eigenvalues of the transformed operator are the same as the eigenvalues of the original operator, and in particular the transformed linearized operator is Fredholm iff the original is Fredholm, the Crandall-Rabinowitz transversality condition holds in one problem iff it holds in the other, and the peigenspaces of the transformed rest points are isomorphic to those of the corresponding rest points in the original problem. Then having proved that bifurcation occurs in the transformed system, this immediately implies bifurcation in the original system. We shall now carry out this program.

For any $\lambda_{0}>0$ consider the boundary condition

$$
\alpha u(x)-\beta \lambda_{0} d u(x) / d n=0, \quad|x|=1 .
$$

Then for $\lambda$ near $\lambda_{0}$, and $k=0,1,2, \ldots$, consider the bounded linear maps $S_{\lambda_{0}}^{\lambda}$ : $C^{k}\left(D^{n}\right) \rightarrow C^{k}\left(D^{n}\right)$, given by

$$
\left(S_{\lambda_{0}}^{\lambda} u\right)(x)=\left[\frac{\alpha}{\beta} \frac{\left(\lambda_{0}-\lambda\right)}{2 \lambda_{0}^{2}}\left(1-|x|^{2}\right)+\frac{\lambda}{\lambda_{0}}\right] u(x) .
$$

An easy calculation shows that if $u$ satisfies the boundary condition $\left(\mathrm{A}_{5}\right)$, then $S_{\lambda_{0}}^{\lambda} u$ satisfies the boundary condition $\left(\mathrm{A}_{6}\right)$. Note that $S_{\lambda_{0}}^{\lambda}$ is equivariant with respect to the action of $O(n)$; i.e., it commutes with $O(n)$ operations. Note too that $S_{\lambda_{0}}^{\lambda_{0}}$ is the identity map, and

$$
S_{\lambda_{0}}^{\lambda} S_{\lambda}^{\lambda_{0}} u(x)=u(x)-\frac{\left(\lambda-\lambda_{0}\right)^{2}}{4 \lambda^{2} \lambda_{0}^{2}} \frac{\alpha^{2}}{\beta^{2}}\left(|x|^{2}-1\right) u(x) .
$$

Thus $S_{\lambda_{0}}^{\lambda}$ is invertible if $\lambda$ is near $\lambda_{0}{ }^{3}$.

${ }^{3}$ More generally, we could consider equation $\left(\mathrm{A}_{4}\right)$ with the boundary conditions $\alpha(\lambda)$. $u(x)+\beta(\lambda) d u(x) / d n=0$ on $|x|=1$, where $\alpha^{2}+\beta^{2}=1$, and $\beta(\lambda) \neq 0$ for all $\lambda$. Then the transformation

$$
S_{\lambda_{0}}^{\lambda} u(x)=\left[\frac{\alpha(\lambda) \beta\left(\lambda_{0}\right)-\beta(\lambda) \alpha\left(\lambda_{0}\right)}{2 \beta\left(\lambda_{0}\right)^{2}}\left(1-|x|^{2}\right)+\frac{\beta(\lambda)}{\beta\left(\lambda_{0}\right)}\right] u(x)
$$

takes functions satisfying the variable (i.e. $\lambda$ ) boundary conditions to the fixed $\lambda_{0}$ boundary conditions. The development we give below, can be carried over immediately to this more general case 
Next, for $k=1,2, \ldots$, let

$$
E^{k}=\left\{(u, \lambda) \in C^{k}\left(D^{n}\right) \times \mathbb{R}: \alpha u(x)-\beta \lambda d u(x) / d n=0,|x|=1\right\},
$$

and define the projection $p: E^{k} \rightarrow \mathbb{R}_{+}$by

$$
p(u, \lambda)=\lambda .
$$

We shall show that $E^{k}$ is a vector bundle over $\mathbb{R}_{+}$, whose fiber at $\lambda$ is $p^{-1}(\lambda)$. Thus, for fixed $\lambda_{0}$, let

$$
F_{\lambda_{0}}^{k}=\left\{u \in C^{k}\left(D^{n}\right): \alpha u(x)-\beta \lambda_{0} d u(x) / d n=0,|x|=1\right\},
$$

and for any $\varepsilon>0$, define a map

$$
S: F_{\lambda_{0}}^{k} \times\left(\lambda_{0}-\varepsilon, \lambda_{0}+\varepsilon\right) \rightarrow p^{-1}\left[\left(\lambda_{0}-\varepsilon, \lambda_{0}+\varepsilon\right] \subset E^{k}\right.
$$

by

$$
S(u, \lambda)=\left(\lambda, S_{\lambda_{0}}^{\lambda} u\right) .
$$

Now $S$ is a vector bundle homomorphism, and for sufficiently small $\varepsilon>0$, it is one-one and onto, so it is an isomorphism. It follows that

$$
p^{-1}\left[\left(\lambda_{0}-\varepsilon, \lambda_{0}+\varepsilon\right)\right] \approx F_{\lambda_{0}}^{k} \times\left(\lambda_{0}-\varepsilon, \lambda_{0}+\varepsilon\right),
$$

so that $p^{-1}\left[\left(\lambda_{0}-\varepsilon, \lambda_{0}+\varepsilon\right)\right]$ is a product, and hence $E^{k}$ is indeed a vector bundle. Note too that $S_{\lambda_{0}}^{\lambda}$ induces an isomorphism between the spaces $F_{\lambda_{0}}^{l}$ and $F_{\lambda}^{l}$ if $\left|\lambda-\lambda_{0}\right|$ is small enough.

Now for $l \in \mathbb{Z}, l \geqq 2$, consider the operator, $M: C^{l} \rightarrow C^{l-2}\left(D^{n}\right)$ defined by

$$
M(u, \lambda)=\Delta\left(u+u_{\lambda}\right)+\lambda^{2} f\left(u+u_{\lambda}\right),
$$

where $\left\{u_{\lambda}: \lambda \in \Lambda\right\}$ is a family of smooth solutions of $\left(\mathrm{A}_{4}\right),\left(\mathrm{A}_{5}\right)$; thus in particular

$$
M(0, \lambda)=0 .
$$

Next, for each fixed $\lambda_{0}$, and each $l \in \mathbb{Z}, l \geqq 2$, for sufficiently small $\varepsilon>0$, define a mapping

$$
L: F_{\lambda_{0}}^{l} \times\left(\lambda_{0}-\varepsilon, \lambda_{0}+\varepsilon\right) \rightarrow C^{l-2}\left(D^{n}\right),
$$

by

$$
L(V, \lambda)=\left(S_{\lambda}^{\lambda_{0}}\right)^{-1} M\left(\lambda, S_{\lambda_{0}}^{\lambda} V\right) .
$$

Notice that solutions of $L=0$ correspond under $S$ in a $1-1$ manner to solutions of $M=0$. Indeed, if $L(V, \lambda)=0$ for some $(V, \lambda)$, then $M S(V, \lambda)=0$, and conversely, if $M(u, \lambda)=0$, then as $(u, \lambda)=S(V, \lambda)$ for some $V \in F_{\lambda_{0}}^{l}$, it follows that $L(V, \lambda)=0$. Finally, observe that since $S_{\lambda_{0}}^{\lambda}$ and $\left(S_{\lambda_{0}}^{\lambda}\right)^{-1}$ are linear, taking derivatives in the $V$-direction gives

$$
d L=\left(S_{\lambda_{0}}^{\lambda}\right)^{-1} d M\left(S_{\lambda_{0}}^{\lambda}\right)
$$


so that $d L$ and $d M$ have the same eigenvalues, and corresponding eigenspaces and kernels. Thus $d L$ is Fredholm iff $d M$ is Fredholm, and the Crandall-Rabinowitz transversality condition, (see [S]), $\left.\frac{d \mu}{d \lambda}\right|_{\lambda=\lambda_{0}} \neq 0$ holds for $d L$ at an eigenvalue $\mu$ iff it holds for $d M$ at $\mu$ because the eigenvalues are identical.

It follows from our above remarks that the rest points of the equation

$$
V_{t}=L(V, \lambda),
$$

correspond in a one-one way to rest points of the equation

$$
u_{t}=M(u, \lambda)
$$

where $\lambda$ is held fixed in both cases. The equation $\left(A_{17}\right)$ corresponds to solutions of $\left(A_{4}\right),\left(A_{5}\right)$. Thus, given any rest point of $\left(A_{17}\right)$, it corresponds to a unique rest point of $\left(A_{16}\right)$, and the peigenspaces of both are isomorphic. Moreover, if $\Phi$ is a Lyapunov function for $\left(\mathrm{A}_{16}\right)$, then $t_{1}>t_{2}$ implies $\Phi\left(V\left(t_{1}\right)\right)>\Phi\left(V\left(t_{2}\right)\right)$, so that $\Phi\left(\left(S_{\lambda_{0}}^{\lambda}\right)^{-1} u\left(t_{1}\right)\right)>\Phi\left(\left(S_{\lambda_{0}}^{\lambda}\right)^{-1} u\left(t_{2}\right)\right)$, and hence $\Phi \circ\left(S_{\lambda_{0}}^{\lambda}\right)^{-1}$ is a Lyapunov function for $\left(\mathrm{A}_{17}\right)$. Since the argument is easily seen to work in the other direction, we see that $\left(A_{16}\right)$ is gradient-like if and only if $\left(A_{17}\right)$ is gradient-like. We can now state the main theorem in this section.

Theorem $\mathbf{A}_{1}$. Assume that $f$ satisfies (4.1), and consider radial solutions $u(\cdot, p)$ of (1.1), (1.2) which lie in the $k^{\text {th }}$ nodal class. Suppose that there are points $p_{1}, p_{2}$, with ${ }^{4} s_{1}<p_{1}<p_{2}<\gamma$ such that the peigenspaces are not isomorphic, $P\left(u\left(\cdot, p_{1}\right)\right)$ $\neq P\left(u\left(\cdot, p_{2}\right)\right)$, and 0 is not in the spectrum of the associated linearized operators about $u\left(\cdot, p_{i}\right), i=1,2$. Then for some $p_{0}, p_{1}<p_{0}<p_{2}, u\left(\cdot, p_{0}\right)$ is a bifurcation point.

Equivalently, we have the following statement.

Theorem $\mathbf{A}_{2}$. Assume that $f$ satisfies (2.1), and that under the change of scale $\left(\mathrm{A}_{3}\right)$, the radial solutions $u_{\lambda_{1}}$ and $u_{\lambda_{2}}$ of $\left(\mathrm{A}_{4}\right),\left(\mathrm{A}_{5}\right)$ satisfy $P_{\lambda_{1}} \neq P_{\lambda_{2}}$, and 0 does not lie in the spectrum of the associated linearized operators about $u_{\lambda_{i}}, i=1,2$. Then there is some $\lambda_{0}, \lambda_{1}<\lambda_{0}<\lambda_{2}$ for which $\left(u_{\lambda_{0}}, \lambda_{0}\right)$ is a bifurcation point.

We shall only give the proof of Theorem $A_{2}$. Note first that since the map $u \rightarrow u+u_{\lambda}$ does not affect the dimensions of the peigenspaces, we see that we may replace problem $\left(\mathrm{A}_{4}\right),\left(\mathrm{A}_{5}\right)$ by the problem $M(\lambda, u)=0$, where $M$ is defined in $\left(\mathrm{A}_{14}\right)$. We are thus studying bifurcation from the trivial solution $u=0$. In these terms the condition $P_{\lambda_{1}} \neq P_{\lambda_{2}}$ becomes

$$
P\left(0, \lambda_{1}\right) \neq P\left(0, \lambda_{2}\right) \text {. }
$$

Suppose first that $\lambda_{2}-\lambda_{1}<\varepsilon$, where $\varepsilon$ is so small that $S_{\lambda_{2}}^{\lambda_{1}}$ is invertible. Then the rest point solutions $\left(0, \lambda_{1}\right),\left(0, \lambda_{2}\right)$ of $\left(A_{14}\right)$ also satisfy $\left(A_{18}\right)$. Since $\left(A_{18}\right)$ is defined on a product space, we may apply Theorem 2.1 (see Lemma $\mathrm{A}_{3}$ ) to conclude the existence of a bifurcation point $\left(0, \lambda_{0}\right), \lambda_{1}<\lambda_{0}<\lambda_{2}$. Now assume that $\lambda_{2}-\lambda_{1}$ is not necessarily small. If $(0, \lambda)$ is not a bifurcation point for each

${ }^{4}$ Recall that $T^{\prime}(p)>0$ if $p>s_{1}$ 
$\lambda \in\left[\lambda_{1}, \lambda_{2}\right]$, then $(0, \lambda)$ is an isolated invariant set for the finite-dimensional equations obtained by our Lyapunov-Schmidt reduction in the proof of Theorem 2.1. By what we have just shown, the Conley index $h^{O(n)}(0, \lambda)$ is locally constant, and hence constant on the entire range $\lambda_{1} \leqq \lambda \leqq \lambda_{2}$. This contradicts $h^{O(n)}\left(0, \lambda_{1}\right)$ $\neq h^{O(n)}\left(0, \lambda_{2}\right)$, and the proof is thus complete.

To conclude these set of ideas, we must show that $\left(\mathrm{A}_{16}\right)$ is a gradient-like system.

Lemma $\mathbf{A}_{3}$. The equation $\left(\mathrm{A}_{17}\right)$, where $u \in E^{k}$ and $\left\{u_{\lambda}\right\}$ is a smooth family of solutions of $\left(\mathrm{A}_{3}\right),\left(\mathrm{A}_{4}\right)$ is a gradient system. In fact, for any fixed $\lambda$, if

$$
\Phi(u, \lambda)=\int_{|x| \leqq 1}\left[\frac{1}{2}\left(u+u_{\lambda}\right) \Delta\left(u+u_{\lambda}\right)+\lambda^{2} F\left(u+u_{\lambda}\right)\right] d x,
$$

where $F^{\prime}=f$, then $d \Phi_{u}=M(u, \lambda)$.

Proof. For $u, w \in E^{k}$,

$$
\begin{aligned}
d \Phi_{u}(w)= & \int_{|x| \leqq 1}\left[\frac{1}{2}\left(u+u_{\lambda}\right) \Delta w\left(u+u_{\lambda}\right)+\frac{1}{2} w \Delta\left(u+u_{\lambda}\right)+\lambda^{2} f\left(u+u_{\lambda}\right) w\right] d x \\
= & \int_{|x| \leqq 1}\left[\Delta\left(u+u_{\lambda}\right)+\lambda^{2} f\left(u+u_{\lambda}\right)\right] w \\
& +\frac{1}{2} \int_{|x|=1}\left[\left(u+u_{\lambda}\right) \frac{d w}{d n}-w \frac{d\left(u+u_{\lambda}\right)}{d n}\right],
\end{aligned}
$$

after integrating twice by parts. But as $u+u_{\lambda}$ and $w$ both satisfy the boundary conditions $\left(A_{5}\right)$, we see that the above boundary integral vanishes. Thus

$$
d \Phi_{u}(w)=\langle M(u, \lambda), w\rangle,
$$

and the proof is complete.

Lemma $\mathbf{A}_{4}$. The equations (c.f. $\left.\left(\mathrm{A}_{4}\right),\left(\mathrm{A}_{5}\right)\right)$,

$$
\begin{aligned}
& u_{t}=\Delta u+\lambda^{2} f(u), \quad(x, t) \in D^{n} \times \mathbb{R}_{+} \\
& \alpha u(x, t)-\beta \lambda d u(x, t) / d n=0, \quad(x, t) \in \partial D^{n} \times \mathbb{R}_{+},
\end{aligned}
$$

form a gradient system, and if

$$
\Phi(u)(t)=\int_{|x| \leqq 1}\left[\frac{1}{2} u \Delta u+\lambda^{2} F(u)\right] d x, \quad\left(F^{\prime}=f\right),
$$

then $d \Phi_{u}=\Delta u+\lambda^{2} f(u)$.

The proof of this result is similar to the last lemma.

\section{References}

[AZ] Amann, H., Zehnder, E.: Nontrivial solutions for a class of nonresonance problems and applications to nonlinear differential equations. Ann. Sc. Norm. Super. Pisa 7, 539-603 (1980) 
[B] Bohme, R.: Die Lösung der Verzweigungsgleichung für nichtlineare Eigenwertprobleme. Math. Z. 127, 105-126 (1972)

[CS] Capell, S., Shaneson, J.: Non-linear similarity. Ann. Math. 113, 315-355 (1981)

[Ch] Chang Kung Ching: Infinite dimensional Morse Theory and its Applications. Sem. Math. Sys., University of Montreal, 1985

[CL] Chow, S.N., Lauterbach, R.: A bifurcation theorem for critical points of variational problems. (Preprint, IMA, Minneapolis, 1985)

[C] Conley, C.: Isolated Invariant Sets and the Morse Index. C.B.M.S., No. 38, Am. Math. Soc.: Providence 1978

[D] Dancer, E.N.: On non-radially symmetric bifurcation. J. Lond. Math. Soc. 20, 287-292 (1979)

[K] Kielhofer, H.: A bifurcation theorem for potential operators. J. Funct. Anal. 77, 1-8 (1988)

[Kr] Krasnosel'skii, M.A.: Topological Methods in the Theory of Nonlinear Integral Equations. Pergamon: Oxford 1964

[L] Lang, S.: Differential Manifolds, Addison-Wesley: Reading, 1972

[LS] Lee, C.N., Wasserman, A.: On the groups $J O(G)$. Mem. Am. Math. Soc., vol. 159, 1975

[P] Pacella, F.: Equivariant Morse theory for flows and an application to the $N$-body problem. Trans. Am. Math. Soc. 297, 41-52 (1986)

[Po] Pospiech, C.: The curious link chain, in Bifurcation: Analysis, Applications, and Algorithms. Proc. Conf. Univ. Dortmund, Aug. 1986, Birkhäuser: Basel Boston Stuttgart, 1987

[Ry] Rybakowski, K.: The Homotopy Index and Partial Differential Equations. Springer: Berlin Heidelberg New York, 1987

[R] Rabinowitz, P.: A bifurcation theorem for potential operators. J. Funct. Anal. 25, 412-424 (1977)

[S] Smoller, J.: Shock Waves and Reaction-Diffusion Equations. Springer: New York Berlin Heidelberg, 1983

[SW $\mathrm{SW}_{1}$ Smoller, J., Wasserman, A.: Existence, uniqueness, and nondegeneracy of positive solutions of semilinear elliptic equations. Commun. Math. Phys. 95, 129-159 (1984)

$\left[\mathrm{SW}_{2}\right]$ Smoller, J., Wasserman, A.: Symmetry-breaking for solutions of semilinear elliptic equations with general boundary conditions. Commun. Math. Phys. 105, 415-451 (1986)

[SW $\mathrm{SW}_{3}$ Smoller, J., Wasserman, A.: Bifurcation from symmetry. Proc. Conf. on Nonlinear Parab. Eqns. (to appear)

[ $\left.\mathrm{SW}_{4}\right]$ Smoller, J., Wasserman, A.: On the monotonicity of the time map. J. Differ. Equations 77, 287-303 (1989)

$\left[\mathrm{SW}_{5}\right]$ Smoller, J., Wasserman, A.: Symmetry, degeneracy, and universality in semilinear elliptic equations J. Funct. Anal. (to appear) 